\title{
Carbon budget and carbon chemistry in Photon Dominated Regions ${ }^{\star}$
}

\author{
D. Teyssier ${ }^{1,2}$, D. Fossé ${ }^{2}$, M. Gerin ${ }^{2}$, J. Pety ${ }^{2,3}$, A. Abergel ${ }^{4}$, and E. Roueff ${ }^{5}$ \\ ${ }^{1}$ Space Research Organization Netherlands, PO Box 800, 9700 AV Groningen, The Netherlands, \\ under an ESA external fellowship \\ 2 Laboratoire d'Étude du Rayonnement et de la Matière, UMR 8112, CNRS, École Normale Supérieure \\ et Observatoire de Paris, 24 rue Lhomond, 75231 Paris Cedex 05, France \\ 3 Institut de Radioastronomie Millimétrique, 300 rue de la Piscine, 38406 St Martin d'Hères, France \\ ${ }^{4}$ Institut d'Astrophysique Spatiale, Université Paris-Sud, Bât. 121, 91405 Orsay Cedex, France \\ 5 LUTH, UMR8102 du CNRS, Observatoire de Paris, Place J. Janssen, 92195 Meudon Cedex, France
}

Received 17 October 2003 / Accepted 9 December 2003

\begin{abstract}
We present a study of small carbon chains and rings in Photon Dominated Regions (PDRs) performed at millimetre wavelengths. Our sample consists of the Horsehead nebula (B33), the $\rho$ Oph L1688 cloud interface, and the cometary-shaped cloud IC 63. Using the IRAM 30-m telescope, the SEST and the Effelsberg 100-m telescope, we mapped the emission of $\mathrm{C}_{2} \mathrm{H}, c-$ $\mathrm{C}_{3} \mathrm{H}_{2}$ and $\mathrm{C}_{4} \mathrm{H}$, and searched for heavy hydrocarbons such as $c-\mathrm{C}_{3} \mathrm{H}, l-\mathrm{C}_{3} \mathrm{H}, l-\mathrm{C}_{3} \mathrm{H}_{2}, l-\mathrm{C}_{4} \mathrm{H}_{2}$ and $\mathrm{C}_{6} \mathrm{H}$. The large scale maps show that small hydrocarbons are present until the edge of all PDRs, which is surprising as they are expected to be easily destroyed by UV radiation. Their spatial distribution reasonably agrees with the aromatic emission mapped in mid-IR wavelength bands. $\mathrm{C}_{2} \mathrm{H}$ and $c-\mathrm{C}_{3} \mathrm{H}_{2}$ correlate remarkably well, a trend already reported in the diffuse ISM (Lucas \& Liszt 2000). Their abundances relative to $\mathrm{H}_{2}$ are relatively high and comparable to the ones derived in dark clouds such as L134N or TMC-1, known as efficient carbon factories. The heavier species are however only detected in the Horsehead nebula at a position coincident with the aromatic emission peak around $7 \mu \mathrm{m}$. In particular, we report the first detection of $\mathrm{C}_{6} \mathrm{H}$ in a PDR. We have run steadystate PDR models using several gas-phase chemical networks (UMIST95 and the New Standard Model) and conclude that both networks fail in reproducing the high abundances of some of these hydrocarbons by an order of magnitude. The high abundance of hydrocarbons in the PDR may suggest that the photo-erosion of UV-irradiated large carbonaceous compounds could efficiently feed the ISM with small carbon clusters or molecules. This new production mechanism of carbon chains and rings could overcome their destruction by the UV radiation field. Dedicated theoretical and laboratory measurements are required to understand and implement these additional chemical routes.
\end{abstract}

Key words. ISM: abundances - astrochemistry - ISM: individual objects: Horsehead nebula, IC 63, $\rho$ Oph

\section{Introduction}

Carbon is the fourth most abundant element in the interstellar medium (ISM), and also the most versatile for building molecules. Carbon chemistry can therefore be considered as the core of interstellar chemistry. Of the nearly 130 molecules now observed in various sources, about $75 \%$ have at least one carbon atom, while one fourth are hydrocarbons. Moreover, the heaviest and most complex molecules are organic molecules with carbon. This statistic does not take into account the Polycyclic Aromatic Hydrocarbons (PAHs), nor the Diffuse Infrared Band carriers (DIB) which are most likely large organic molecules (Herbig 1995). Carbon, neutral or ionized,

Send offprint requests to: D. Teyssier,

e-mail: teyssier@sron.rug.nl

* Appendix A is only available in electronic form at http://www. edpsciences.org is also one of the main reactants in interstellar chemistry networks, due the large number of organic molecules, but also to its versatility allowing it to participate to numerous chemical reactions at any temperature, from the very cold dense cores, to warm and hot gas. Therefore understanding the carbon chemistry is of major importance for astrochemistry, and for star formation.

A large fraction of the chemical reactions in ISM networks involve the numerous hydrocarbons present in the ISM. Such molecules were first reported in circumstellar shells, where the chemistry is particularly favorable to their formation $\left(\mathrm{C}_{4} \mathrm{H}-\right.$ Guélin et al. 1978, $\mathrm{C}_{5} \mathrm{H}-$ Cernicharo et al. 1986), but also in molecular dark clouds, where they are efficiently shielded from the interstellar radiation $\left(\mathrm{C}_{3} \mathrm{H}_{2}-\right.$ Thaddeus et al. 1981, $\mathrm{C}_{2} \mathrm{H}$ - Gottlieb et al. 1983). Since then, hydrocarbons of increasingly greater number of carbons have been reported in several 
objects (e.g. $\mathrm{C}_{6} \mathrm{H}-$ Guélin et al. 1987, $\mathrm{C}_{8} \mathrm{H}-$ Dickens et al. 2001). In the diffuse gas, molecules like $\mathrm{CH}, \mathrm{CH}^{+}$and $\mathrm{CN}$ are known since the 40 's, and carbon clusters $\left(\mathrm{C}_{2}, \mathrm{C}_{3}\right)$ are now almost routinely detected in the visible towards bright stars (Maier et al. 2001; Roueff et al. 2002; Oka et al. 2003) as well as in the far-IR (e.g. Cernicharo et al. 2000). Using radio telescopes, Lucas \& Liszt (2000) have also shown that $\mathrm{C}_{2} \mathrm{H}$ and $c-\mathrm{C}_{3} \mathrm{H}_{2}$ are ubiquitous in diffuse gas, confirming previous work by Cox et al. (1989).

A natural question therefore arises: if carbon chains are present in the diffuse ISM, what does happen in the PhotonDominated Regions (hereafter PDRs)? As in diffuse clouds, the chemical processes are dominated by the radiation but the gas is denser. The radiation field can also be constrained (intensity and direction) and, with the new data obtained by ISO, an accurate picture of the mid-IR emission due to the Aromatic Infrared Band (AIB) carriers at these cloud interfaces has emerged. Knowledge about the distribution of carbon chain and rings in PDRs is however yet scarce and limited to the works of Fuente et al. (1993, 2003), who reported the observation of $\mathrm{C}_{2} \mathrm{H}$ at some positions in NGC 7023, the Orion Bar and NGC 7027, and Ungerechts et al. (1997), who presented maps of the Orion Bar in the millimetre transitions of $\mathrm{C}_{2} \mathrm{H}$ and cyclic $-\mathrm{C}_{3} \mathrm{H}_{2}$. In this paper we extend the study of hydrocarbons in PDRs through an extensive inventory of carbon chains and rings of up to six carbon atoms. Our aims are to get a better view of their distribution on large scale with respect to other standard tracers, and to derive their contribution to the total carbon budget in comparison with values measured in other regions.

The paper is organised as follows. We present in Sect. 2 the three sources studied here, while the observations are described in Sect. 3. We then analyse the spatial distribution of the mapped hydrocarbons (Sect. 4.1) and derive the molecular column densities of the observed species (Sect. 5.1). These results are compiled in the form of a carbon budget and compared to similar observations reported in dark clouds and in the diffuse ISM (Sects. 5.2 and 6.2). We finally compare the inferred abundances to numerical PDR models (Sect. 6.3) and present our conclusions in Sect. 7.

\section{Presentation of the sources}

\subsection{The Horsehead nebula}

In the visible, the Horsehead nebula (B33, Barnard 1919) appears as a dark patch seen in silhouette against the ionized $\mathrm{H} \alpha$ emission emanating from the IC434 HII region. The large-scale maps reported by e.g. Maddalena et al. (1986, $\mathrm{CO}(2-1))$ or Lada et al. (1991, CS) show its connection to the L1630 cloud, which belongs to the Orion B molecular complex (see e.g. Abergel et al. 2002 for an overview of the region). In the scenario proposed by Reipurth \& Bouchet (1984), the Horsehead nebula is interpreted as an early Bok globule emerging from its parent cloud via the eroding incident radiation field emitted by the close-by $09.5 \mathrm{~V}$ star $\sigma$ Ori. The Horsehead nebula thus corresponds to a condensation resisting the illumination and it presents on its western border a PDR whose detailed

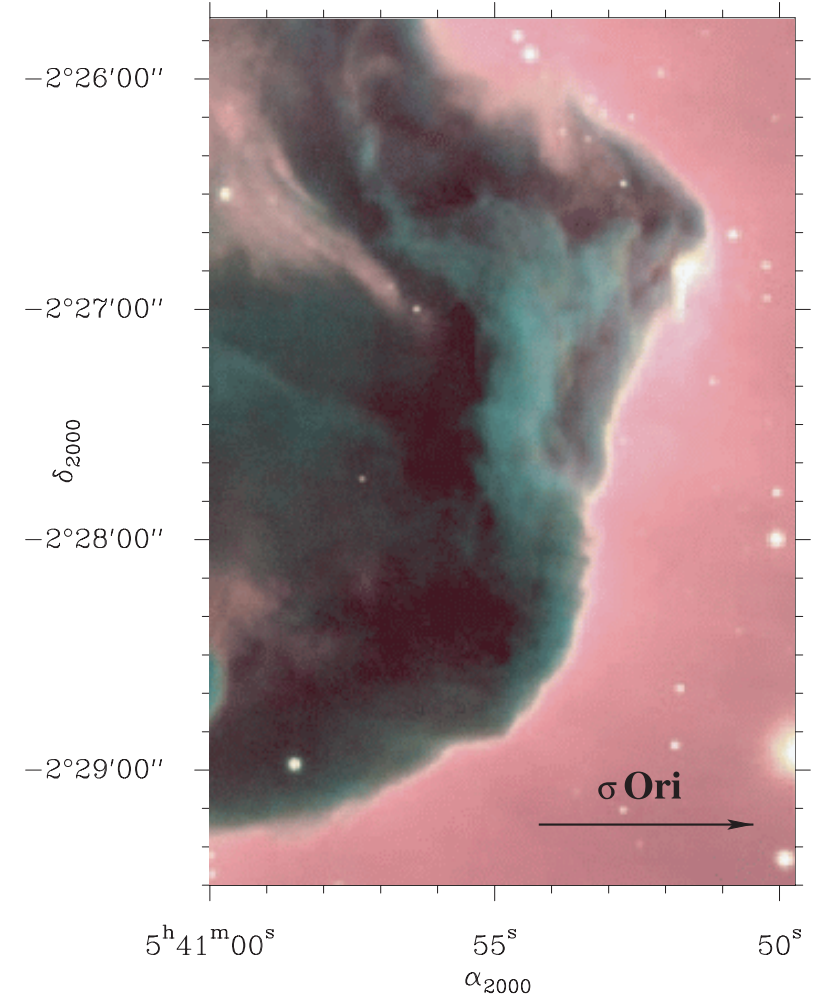

Fig. 1. Composite colour image of the Horsehead nebula PDR obtained by the VLT in the $B, V$ and $R$ bands. The arrow indicates the direction of the illuminating star $\sigma$ Ori (Image courtesy of ESO).

shape is displayed in Fig. 1. Assuming a distance of $400 \mathrm{pc}$ (Anthony-Twarog 1982), and considering a projected distance to $\sigma$ Ori of $0.5^{\circ}$, the incident radiation field is of order $G_{0}=100$ (Zhou et al. 1993; Abergel et al. 2003). This can be seen as relatively weak compared to other well-known PDRs of the solar neighborhood (e.g. the Orion Bar, NGC 7023). This PDR was mapped by ISO ( 6 " resolution) in filters around 7 and $15 \mu \mathrm{m}$ (Abergel et al. 2002, 2003). It reveals a very thin filament (10", or $\sim 0.02 \mathrm{pc}$ at $400 \mathrm{pc}$ ) whose narrow size suggests that the PDR is seen perfectly edge-on (see also Fig. 4).

Little attention was paid to this object until recently. In the CO and isotope maps of Kramer et al. (1996), the nebula is heavily diluted in the $2^{\prime}$ beam and only appears as a small intrusion of L1630 into the HII region. These large-scale data already indicated mean densities of order $3 \times 10^{4} \mathrm{~cm}^{-3}$ (Zhou et al. 1993) and column densities compatibles with $A_{\mathrm{V}} \sim 3$ (Kramer et al. 1996). Observations at higher spatial resolution $\left(10-20^{\prime \prime}\right)$ were only reported very recently. Pound et al. (2003) presented BIMA $\mathrm{CO}(1-0)$ and discussed in details the possible origin and evolution of the dark cloud. They reveal a complex velocity structure but their data are likely affected by nonnegligible optical depth effects. Abergel et al. (2003) report IRAM 30-m maps obtained in the $(J=1-0)$ and $(J=2-1)$ transitions of $\mathrm{CO},{ }^{13} \mathrm{CO}$ and $\mathrm{C}^{18} \mathrm{O}$. They infer densities compatible with the values reported at larger scale, and show clear selective photo-dissociation of the considered species at the PDR edge. 


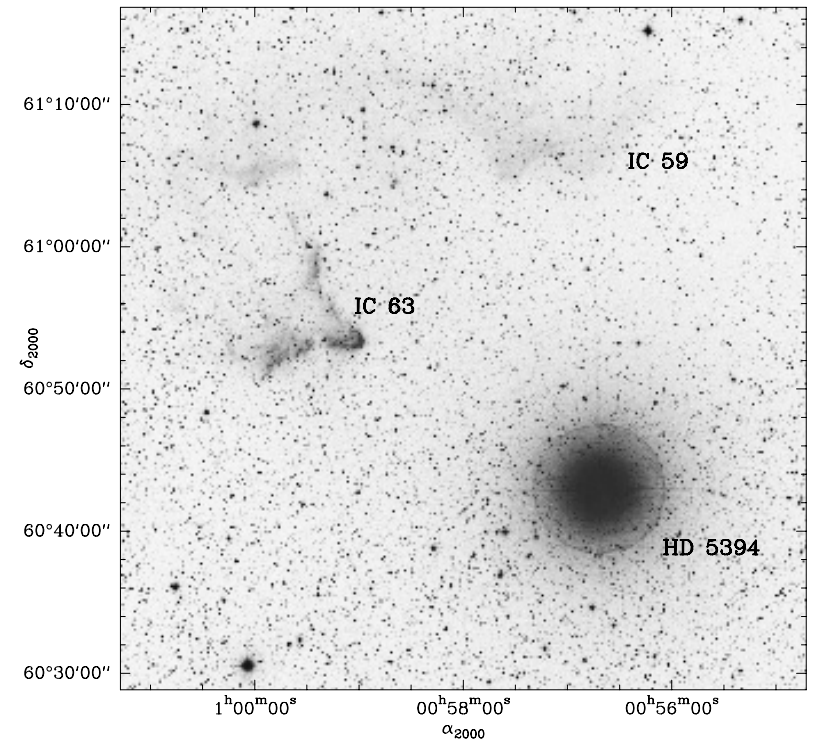

Fig. 2. Visible image from the Digital Sky Survey in the area of IC 63. The cometary shape originates from the strong radiation field emitted by the close-by HD5394 stars 20' South-West.

\subsection{IC 63}

IC 63 is a reflexion nebula associated with the B0.5IVpe variable star $\gamma$ Cas (HD5394, $\left.G_{0}=1100\right)$ located $230 \pm 70 \mathrm{pc}$ from us (Vakili et al. 1984). This strong radiation field has created a PDR at the western border of the cometary-shaped molecular cloud reported by Jansen et al. (1994). IC 63 is associated to another reflexion nebula, namely IC 59, located at a projected distance of $20^{\prime}$ (or $1.3 \mathrm{pc}$ ), as is illustrated in Fig. 2. According to Blouin et al. (1997), IC 59 would be lying in the background of the IC 63-HD5394 pair. Jansen et al. (1994), on the other hand, claim that the illuminating star would be closer to the observer, so that IC 63 is partially seen face-on.

The molecular component appears elongated and seems well aligned with the direction of $\gamma$ Cas. Jansen et al. (1994) report that the $\mathrm{CO}$ and $\mathrm{CS}$ emissions are confined into a cometary cloud of size $\sim 1^{\prime} \times 2^{\prime}$, and assume an even smaller area of $30^{\prime \prime} \times 20^{\prime \prime}$ for all other species than CO. This has particular consequences in terms of beam dilution. These authors derive $T_{\text {kin }}=50 \pm 10 \mathrm{~K}$ and $n_{\mathrm{H}_{2}}=5 \pm 2 \times 10^{4} \mathrm{~cm}^{-3}$, with a possible density gradient along the cloud major axis. The inferred $\mathrm{H}_{2}$ column densities translate into $A_{\mathrm{V}}=6.3 \pm 2.5 \mathrm{mag}$. Finally, an analysis of the carbon budget in this source indicates that the total gas phase carbon abundance $\left(5.4 \times 10^{-5}\right)$ is only $13 \%$ of the solar one, which suggests that the bulk of the carbon is in solid phase and might be located on PAHs or larger grains.

IC 63 has also been mapped with ISOCAM in the CVF mode allowing imaging spectroscopy between 5 and $16.5 \mu \mathrm{m}$ (see Fossé et al. 2000). Figure 5 shows the emission observed around the $6.2 \mu \mathrm{m}$ AIB feature, which corresponds to a stretching mode of the $\mathrm{C}-\mathrm{C}$ bond. The aromatic emission follows the PDR border and exhibit several peaks just behind the PDR and inside the molecular tail.

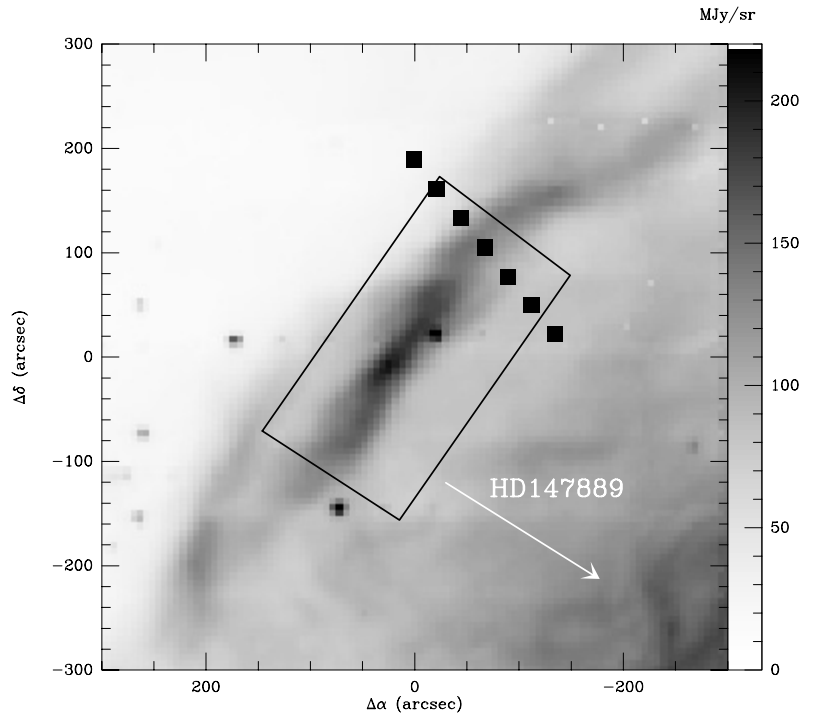

Fig. 3. Close-up of the western border of L1688 mapped by ISOCAM around $7 \mu \mathrm{m}$ (Abergel et al. 1996). The illuminating star HD 147889 direction is indicated by an arrow. The black squares indicate the positions of the cut where molecular data have been collected. The tilted rectangle delimitates the area mapped in ${ }^{13} \mathrm{CO}$ and $\mathrm{C}^{18} \mathrm{O}$. Offsets are given with respect to $\alpha_{2000}=16^{\mathrm{h}} 25^{\mathrm{m}} 58^{\mathrm{s}}, \delta_{2000}=-24^{\circ} 21^{\prime} 00^{\prime \prime}$.

\subsection{The $\rho$ Ophiuchi L1688 cloud}

The Ophiuchi molecular complex is a close-by star formation region (distance $135 \pm 15$ pc, Habart et al. 2003). While its socalled "North" area is considered inactive, the West counterpart contains two main massive cores, L1688 and L1689, heated on large scales by the Sco-OB2 association. We concentrate here on the L1688 complex, which hosts numerous pre-stellar cores (e.g. Motte et al. 1998), as well as hundreds of YSOs (Greene \& Young 1992; Bontemps et al. 2001), very likely responsible for the strong luminosity in the infrared.

ISOCAM observations revealed bright interlaced filaments of width $\sim 0.03 \mathrm{pc}$ coinciding with the western border of the cloud (Fig. 3, see also Abergel et al. 1996). It corresponds to a PDR (hereafter called L1688-W) illuminated by the B2V star HD147889. According to Abergel et al. (1999), this star is located in the centre of a spherical cavity exhibiting irregular edges. The filamentary structure is interpreted as the edge-on regions of this cavity. Assuming that the star and PDRs are in a common plane perpendicular to the line of sight, Habart et al. (2003) infer an incident radiation field of $G_{0}=400$. Based on the AIB and the $\mathrm{H}_{2}(1-0) \mathrm{S}(1)$ line emissions, these authors predict a density plateau of $n_{\mathrm{H}}=4 \times 10^{4} \mathrm{~cm}^{-3}$ inside the cloud, followed by a decrease of the form $n_{\mathrm{H}}(r) \propto r^{2.5}$ towards the radiating star, where $r$ is the radial dimension across the PDR. This drop occurs at $\Delta \alpha \sim-100^{\prime \prime}$ on the cut indicated in Fig. 3.

On large scales, the mid-IR emission shape is well reproduced by the $\mathrm{CO}$ and isotopes maps obtained by Lada \& Wilking (1980) and Wilking \& Lada (1983). Their data revealed a complex velocity structure and, in particular, significant self-absorption in the ${ }^{13} \mathrm{CO}$ species. On these scales, ${ }^{12} \mathrm{CO}$ data are compatible with $A_{\mathrm{V}}$ in the range 50-100 mag. 


\section{Observations}

The data presented in this paper have been gathered between September 1999 and May 2002 at the IRAM 30-m, the CSO, the SEST and the Effelsberg 100- $\mathrm{m}^{1}$ telescopes. They are concentrated on a series of molecules including $\mathrm{CO}$ and its main isotopomers, as well as a collection of small carbon chains and rings. The details on the considered lines are compiled in Table 1 . We give here some additional information for each source. In the following the prefixes $c$ - and $l$ - will respectively refer to the cyclic and linear isomers of the considered species.

\subsection{Horsehead nebula}

The Horsehead nebula PDR was mapped at the 30-m telescope in the $(J=1-0)$ and $(J=2-1)$ transitions of $\mathrm{C}^{18} \mathrm{O}$, as well as in $c-\mathrm{C}_{3} \mathrm{H}_{2}\left(2_{1,2}-1_{0,1}\right), \mathrm{C}_{2} \mathrm{H}(1-0)$ and $\mathrm{C}_{4} \mathrm{H}(9-8)$ (Fig. 4). System temperatures at $3 \mathrm{~mm}$ were in the range $100-120 \mathrm{~K}$. Some weaker lines were probed at dedicated positions (Figs. 8 and 9) in $\mathrm{CS}, c-\mathrm{C}_{3} \mathrm{H}\left(2_{1,2}-1_{1,1}\right), l-\mathrm{C}_{3} \mathrm{H}(9 / 2-$ $7 / 2), l-\mathrm{C}_{3} \mathrm{H}_{2}\left(3_{3,0}-2_{2,1}\right), l-\mathrm{C}_{4} \mathrm{H}_{2}\left(11_{0,11}-10_{0,10}\right), \mathrm{HN}^{13} \mathrm{C}(1-0)$, $\mathrm{CH}_{3} \mathrm{CCH}\left(5_{k}-4_{k}\right)$ and $\mathrm{C}_{6} \mathrm{H}(59 / 2-57 / 2)$. Except from the on-thefly maps of $\mathrm{C}^{18} \mathrm{O}$, all spectra were obtained in the Frequency Switching mode using an autocorrelator spectrometer providing $80 \mathrm{kHz}$ resolution elements. Additional data were obtained in $\mathrm{C}_{2} \mathrm{H}(3-2)$ at the CSO (AOS spectrometer, $T_{\text {sys }} \sim 800 \mathrm{~K}$ ), and in $l-\mathrm{C}_{3} \mathrm{H}_{2}\left(1_{0,1}-0_{0,0}\right)$ and $c-\mathrm{C}_{3} \mathrm{H}_{2}\left(1_{1,0}-1_{0,1}\right)$ at the Effelsberg $100-\mathrm{m}$ telescope $\left(T_{\text {sys }} \sim 90 \mathrm{~K}\right)$. Moreover, a $1.2 \mathrm{~mm}$ continuum emission map was obtained at the 30-m using the new 117-channel MAMBO bolometer (11" resolution). Using a fast-mapping mode (Teyssier \& Sievers 1999), we could cover areas of $8.5^{\prime} \times 7.5^{\prime}$ in about $30 \mathrm{~min}$, with different chopping throws and at different hour angles, reducing efficiently some mapping artifacts inherent to the EKH inversion technique applied here (Emerson et al. 1979). Only part of this map is displayed here (Fig. 4) and further results from these data will be discussed in a forthcoming paper.

The data were first calibrated to the $T_{\mathrm{A}}^{*}$ scale using the socalled chopper wheel method (Penzias \& Burrus 1973). The final adopted scale however depends on the source size. In the particular case of the Horsehead nebula, we applied the correction factor introduced in Abergel et al. (2003) taking into account the error beam of the $30-\mathrm{m}$ telescope. At $3 \mathrm{~mm}$, we assumed hydrocarbon emission areas similar to the $\mathrm{C}^{18} \mathrm{O}$ one.

\subsection{IC 63}

IC 63 was mapped at the $30-\mathrm{m}$ in $\mathrm{CO}(2-1)$ and $\mathrm{C}_{2} \mathrm{H}(1-0)$ using the on-the-fly mode (Fig. 5). Orthogonal coverages were optimally combined using the PLAIT algorithm by Emerson \& Graeve (1988). In addition to $\mathrm{C}^{18} \mathrm{O}(2-1)$, the same lines as in the Horsehead were probed along a cut crossing the PDR (see Fig. 9). All other observing parameters were similar to the Horsehead ones. Dedicated positions were also observed at the 100 - $\mathrm{m}$ in $c-\mathrm{C}_{3} \mathrm{H}_{2}\left(1_{1,0}-1_{0,1}\right)$.

\footnotetext{
1 The 100-m telescope is operated by the MPIfR (Max-PlanckInstitut für Radioastronomie).
}

Table 1. Line parameters for the all the species and data reported in this paper. When two spatial resolutions are indicated, we refer to the $H B P W$ 's corresponding to the IRAM 30-m and the SEST telescopes respectively. ${ }^{\left({ }^{a}\right)}$ Observed at the CSO. ${ }^{\left({ }^{b}\right)}$ Observed at the Effelsberg 100-m telescope. See Sect. 3 for details.

\begin{tabular}{|c|c|c|c|}
\hline Molecule & Transition & $\begin{array}{c}\text { Frequency } \\
(\mathrm{GHz})\end{array}$ & $\begin{array}{l}H P B W \\
(\operatorname{arcsec})\end{array}$ \\
\hline${ }^{12} \mathrm{CO}$ & $J=2-1$ & 230.538000 & 11 \\
\hline${ }^{13} \mathrm{CO}$ & $J=2-1$ & 220.398686 & $22 / 44$ \\
\hline \multirow[t]{2}{*}{$\mathrm{C}^{18} \mathrm{O}$} & $J=1-0$ & 109.782160 & $22 / 44$ \\
\hline & $J=2-1$ & 219.560357 & 11 \\
\hline $\mathrm{CS}$ & $J=2-1$ & 97.980950 & 24 \\
\hline \multirow[t]{12}{*}{$\mathrm{C}_{2} \mathrm{H}$} & $N=1-0, J=3 / 2-1 / 2$ & & \\
\hline & $F=2-1$ & 87.316925 & $28 / 56$ \\
\hline & $F=1-0$ & 87.328624 & $28 / 56$ \\
\hline & $N=1-0, J=1 / 2-1 / 2$ & & \\
\hline & $F=1-1$ & 87.402004 & $28 / 56$ \\
\hline & $\begin{array}{c}F=0-1 \\
\end{array}$ & 87.407165 & $28 / 56$ \\
\hline & $N=3-2, J=7 / 2-5 / 2$ & & \\
\hline & $F=4-3$ & 262.004260 & $28^{(a)}$ \\
\hline & $F=3-2$ & 262.006480 & $28^{(a)}$ \\
\hline & $N=3-2, J=5 / 2-3 / 2$ & & \\
\hline & $F=3-2$ & 262.064990 & $28^{(a)}$ \\
\hline & $F=2-1$ & 262.067460 & $28^{(a)}$ \\
\hline \multirow[t]{6}{*}{$c-\mathrm{C}_{3} \mathrm{H}$} & $N=2_{12}-1_{11}, J=5 / 2-3 / 2$ & & \\
\hline & $F=3-2$ & 91.494349 & $27 / 54$ \\
\hline & $F=2-1$ & 91.497608 & $27 / 54$ \\
\hline & $N=2_{12}-1_{11}, J=3 / 2-1 / 2$ & & \\
\hline & $F=1-0$ & 91.692752 & $27 / 54$ \\
\hline & $F=2-1$ & 91.699471 & $27 / 54$ \\
\hline \multirow[t]{5}{*}{$l-\mathrm{C}_{3} \mathrm{H}$} & $J=9 / 2-7 / 2$ & & \\
\hline & $F=5-4(\mathrm{f})$ & 97.995166 & $25 / 50$ \\
\hline & $F=4-3(\mathrm{f})$ & 97.995913 & $25 / 50$ \\
\hline & $F=5-4(\mathrm{e})$ & 98.011611 & $25 / 50$ \\
\hline & $F=4-3(\mathrm{e})$ & 98.012524 & $25 / 50$ \\
\hline \multirow[t]{2}{*}{$c-\mathrm{C}_{3} \mathrm{H}_{2}$} & $1_{1,0}-1_{0,1}$ & 18.343145 & $54^{(b)}$ \\
\hline & $2_{1,2}-1_{0,1}$ & 85.338898 & $28 / 56$ \\
\hline \multirow[t]{2}{*}{$l-\mathrm{C}_{3} \mathrm{H}_{2}$} & $1_{0,1}-0_{0,0}$ & 20.792590 & $48^{(b)}$ \\
\hline & $3_{3,0}-2_{2,1}$ & 216.27875 & 11 \\
\hline \multirow[t]{6}{*}{$\mathrm{C}_{4} \mathrm{H}$} & $N=9-8, J=19 / 2-17 / 2$ & & \\
\hline & $F=9-8$ & 85.634006 & $28 / 56$ \\
\hline & $F=10-9$ & 85.634017 & $28 / 56$ \\
\hline & $N=9-8, J=17 / 2-15 / 2$ & & \\
\hline & $F=8-7$ & 85.672581 & $28 / 56$ \\
\hline & $F=8-8$ & 85.672583 & $28 / 56$ \\
\hline$l-\mathrm{C}_{4} \mathrm{H}_{2}$ & $11_{0,11}-10_{0,10}$ & 98.245016 & 24 \\
\hline \multirow[t]{4}{*}{$\mathrm{CH}_{3} \mathrm{C}_{2} \mathrm{H}$} & $J=5-4$ & & \\
\hline & $K=2$ & 85.450730 & 28 \\
\hline & $K=1$ & 85.455622 & 28 \\
\hline & $K=0$ & 85.457272 & 28 \\
\hline \multirow[t]{2}{*}{$\mathrm{C}_{6} \mathrm{H}$} & ${ }^{2} \Pi_{3 / 2} J=59 / 2-57 / 2 \mathrm{e}$ & 81.777893 & 28 \\
\hline & ${ }^{2} \Pi_{3 / 2} J=59 / 2-57 / 2 \mathrm{f}$ & 81.801247 & 28 \\
\hline \multirow[t]{4}{*}{$\mathrm{HN}^{13} \mathrm{C}$} & $J=1-0$ & & \\
\hline & $F=0-1$ & 87.090735 & 27 \\
\hline & $F=2-1$ & 87.090859 & 27 \\
\hline & $F=1-1$ & 87.090942 & 27 \\
\hline $\mathrm{HC}_{3} \mathrm{~N}$ & $J=9-8$ & 81.881468 & 28 \\
\hline
\end{tabular}




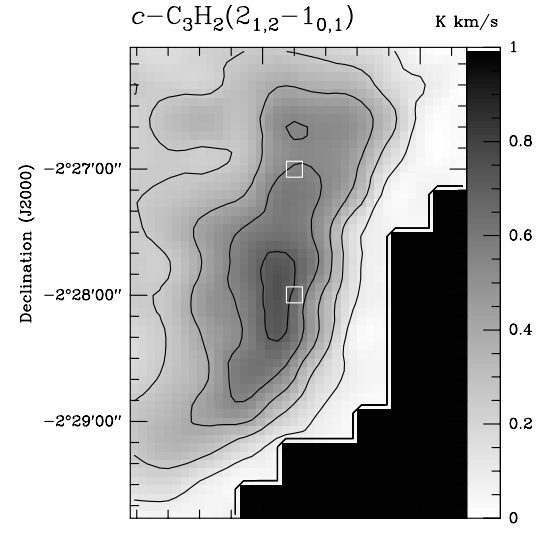

ISOCAM $5.5-8.5 \mu \mathrm{m} \quad \mathrm{MJy} / \mathrm{sr}$

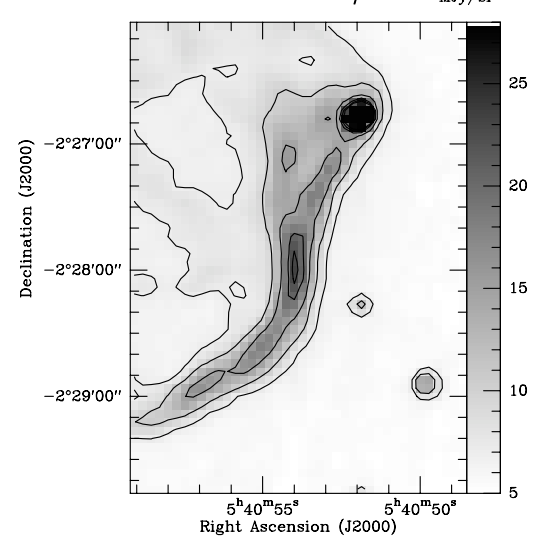

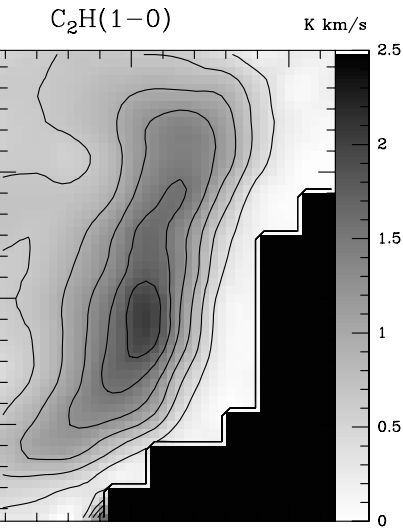
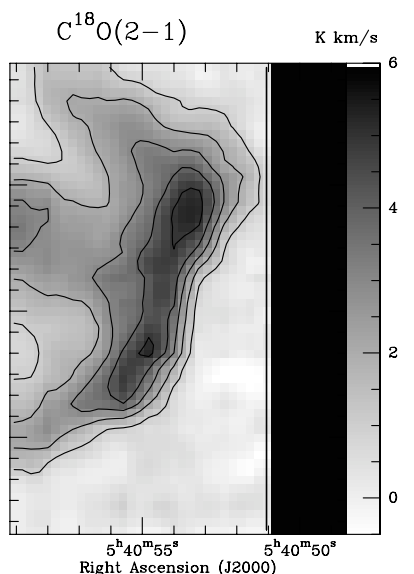

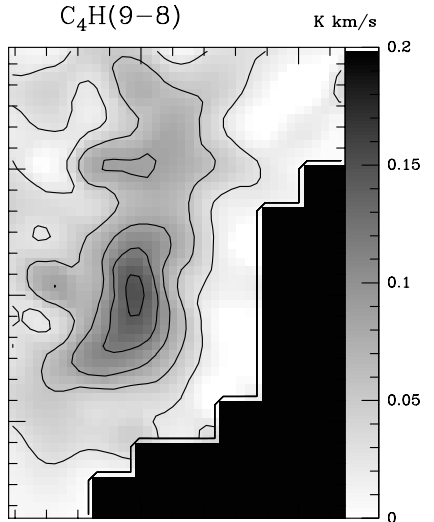

$1.2 \mathrm{~mm}$ continuum $\mathrm{mJy} / \mathrm{beam}$

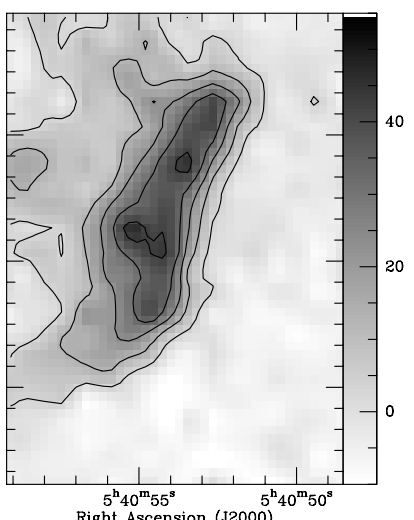

Fig. 4. Integrated emission maps $\left(T_{\mathrm{A}}^{*}, \mathrm{~K} \mathrm{~km} \mathrm{~s}^{-1}\right)$ of the molecular emission in the Horsehead nebula PDR for several species. Contours are: $c-\mathrm{C}_{3} \mathrm{H}_{2}\left(2_{1,2}-1_{0,1}\right) 0.1$ to 0.7 by $0.15, \mathrm{C}_{2} \mathrm{H}(1-0) 0.3$ to 2.1 by $0.3, \mathrm{C}_{4} \mathrm{H}(9-8) 0.025$ to 0.2 by $0.03, \mathrm{C}^{18} \mathrm{O}(2-1) 1$ to 6 by 1 . Also displayed is the continuum emission between 5 and $8.5 \mu \mathrm{m}$ (aromatic emission) as mapped by ISO (from Abergel et al. 2002, contours are 10 to 25 by $5 \mathrm{MJy} / \mathrm{sr}$ ), and at $1.2 \mathrm{~mm}$ (contours are 3 to 53 by $10 \mathrm{mJy} / 11^{\prime \prime}$ beam) mapped by the MAMBO 117-channel bolometer. The white squares indicated the position labelled as CO-peak (top) and IR-peak (bottom).

After calibrating to the $T_{\mathrm{A}}^{*}$ scale, the lack of large-scale information in the probed molecules motivated us to adopt the widely-used main beam brightness temperature $T_{\mathrm{mb}}$ assuming beam efficiencies at the IRAM 30-m of $\eta_{\mathrm{b}}\left(=B_{\text {eff }} / F_{\text {eff }}\right.$ in the IRAM nomenclature $)=0.79$ and 0.49 at $3 \mathrm{~mm}$ and $1.3 \mathrm{~mm}$ respectively. An additional correction was considered to account for the source dilution in the beam (see the description by Jansen et al. 1994) applying deconvolved sizes as inferred in Sect. 4.3.

\section{3. $L 1688-W$}

The L1688-W cloud was observed at the SEST in July 2000. We mapped the ${ }^{13} \mathrm{CO}(2-1)$ and $\mathrm{C}^{18} \mathrm{O}(1-0)$ line emissions in an area indicated in Fig. 3. The area we have covered is just at the edge of the large-scale map of Wilking \& Lada (1983). This figure also shows the position of the cut performed in Frequency Switching for some of the small hydrocarbon species listed above. Figure 6 displays the integrated intensity maps of the two CO isotopomers. The corresponding observing parameters can be found in Table 1.

As for IC 63, the data were finally scaled to main beam brightness temperature, assuming SEST beam efficiencies of $\eta_{\mathrm{b}}=0.78$ and 0.56 at $3 \mathrm{~mm}$ and $1.3 \mathrm{~mm}$ respectively.

\section{Results}

\subsection{General trends}

Despite the strong incident radiation fields $\left(G_{0} \sim 100-1000\right)$ compared to the diffuse ISM $\left(G_{0} \sim 1\right)$, the small carbon chains and rings are detected in all objects. At first glance, this is surprising as small hydrocarbons are expected to be rapidly destroyed by such UV radiations. The spatial intensity variations of all hydrocarbons measured in this study correlate well. This result was already reported by Lucas \& Liszt (2000) in the diffuse ISM for $c-\mathrm{C}_{3} \mathrm{H}_{2}$ and $\mathrm{C}_{2} \mathrm{H}$. Figure 7 illustrates this trend in the Horsehead nebula. Some additional information is given below for each of the sources.

\subsection{Horsehead nebula}

The maps obtained in the Horsehead nebula are displayed in Fig. 4. They show that the hydrocarbon large-scale distribution correlates well with the PDR structure as traced by ISO or ${ }^{12} \mathrm{CO}$ (see maps by Abergel et al. 2003). In particular the sharp edge, although smoothed by the beams, is observed in all tracers.

On smaller scales, different behaviours have to be mentioned. It is indeed interesting to note the different positions of the respective hydrocarbon and $\mathrm{CO}$ (and isotopomers) peaks. 

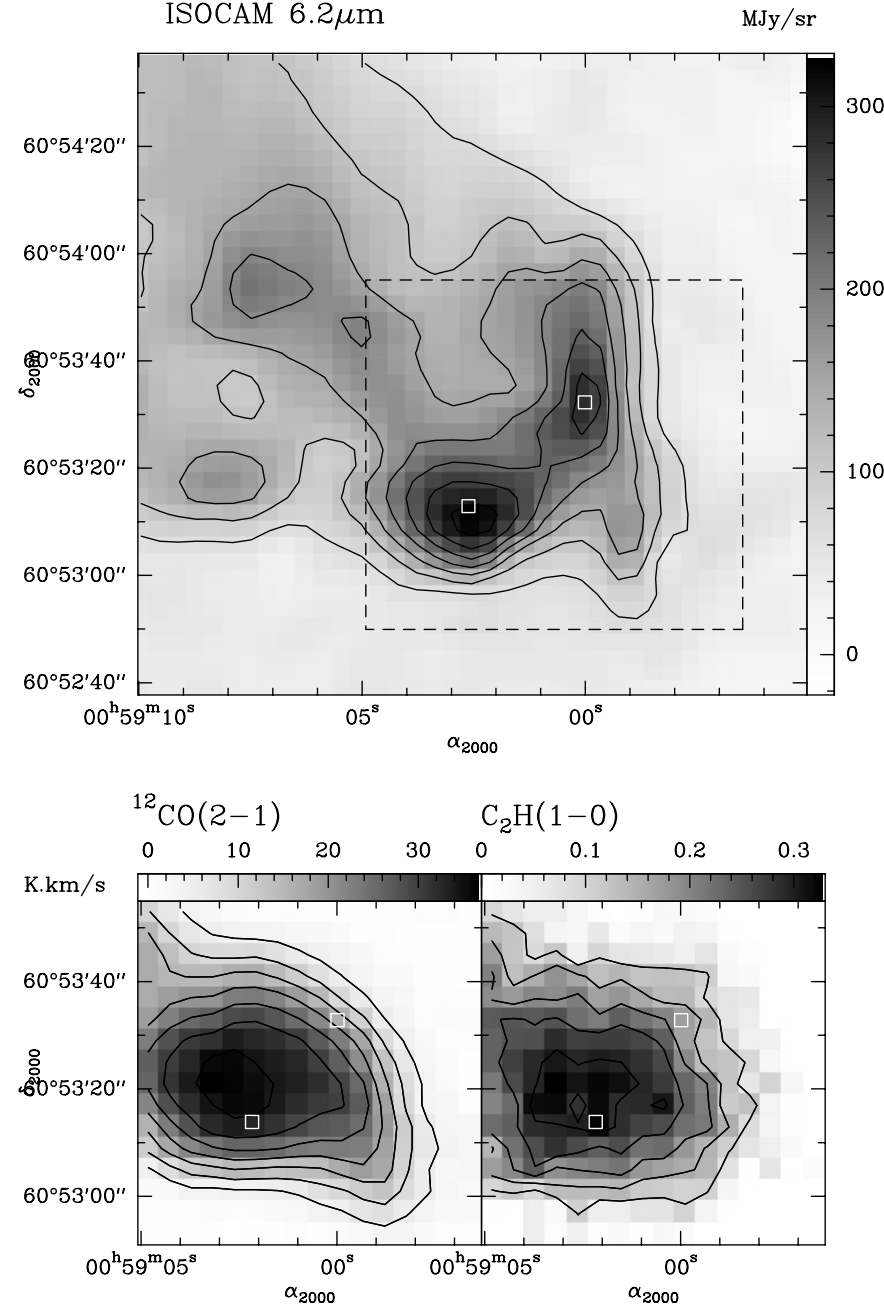

Fig. 5. Maps of IC 63. Top: integrated emission of the ISOCAM CVF spectra between 5.98 and $6.54 \mu \mathrm{m}$ after subtraction of a 1-order baseline. Contours are 70 to 310 by $40 \mathrm{MJy} / \mathrm{sr}$. The dashed box indicates the area covered by the molecular millimetre data displayed below. Bottom: integrated emission maps $\left(T_{\mathrm{A}}^{*}\right.$, $\mathrm{K} \mathrm{km} \mathrm{s}^{-1}$ ) of ${ }^{12} \mathrm{CO}(2-1)$ (contours 5 to 40 by 5 ) and $\mathrm{C}_{2} \mathrm{H}(1-0)$ (contours 0.10 to 0.35 by 0.05 ). The white squares indicate the position of the IR peaks.

Figure 7 shows that the correlation between $\mathrm{C}^{18} \mathrm{O}$ and $\mathrm{C}_{2} \mathrm{H}$ is worse than with $c-\mathrm{C}_{3} \mathrm{H}_{2}$, though the former lines are the strongest and thus offer better signal-to-noise ratio. Moreover, the hydrocarbon peak is located very close to the peak of aromatic emission around $7 \mu \mathrm{m}$ (hereafter called IR peak), suggesting a chemical link between AIB carriers and the small hydrocarbons. More conclusive comparisons with the ISO mid-IR data are nevertheless limited by the 30-m spatial resolution. This has important consequences on the physicochemical analysis along cuts crossing the PDR as, for instance, any abundances probed at the IR peak will not coincide with the peak emission of the hydrocarbons. This issue will be addressed in a companion paper presenting interferometric observations of the same molecules (resolution $\sim 5^{\prime \prime}$, Pety et al. 2003).

Other species were also probed at positions of interest. They include heavier and rarer hydrocarbons, as well as some

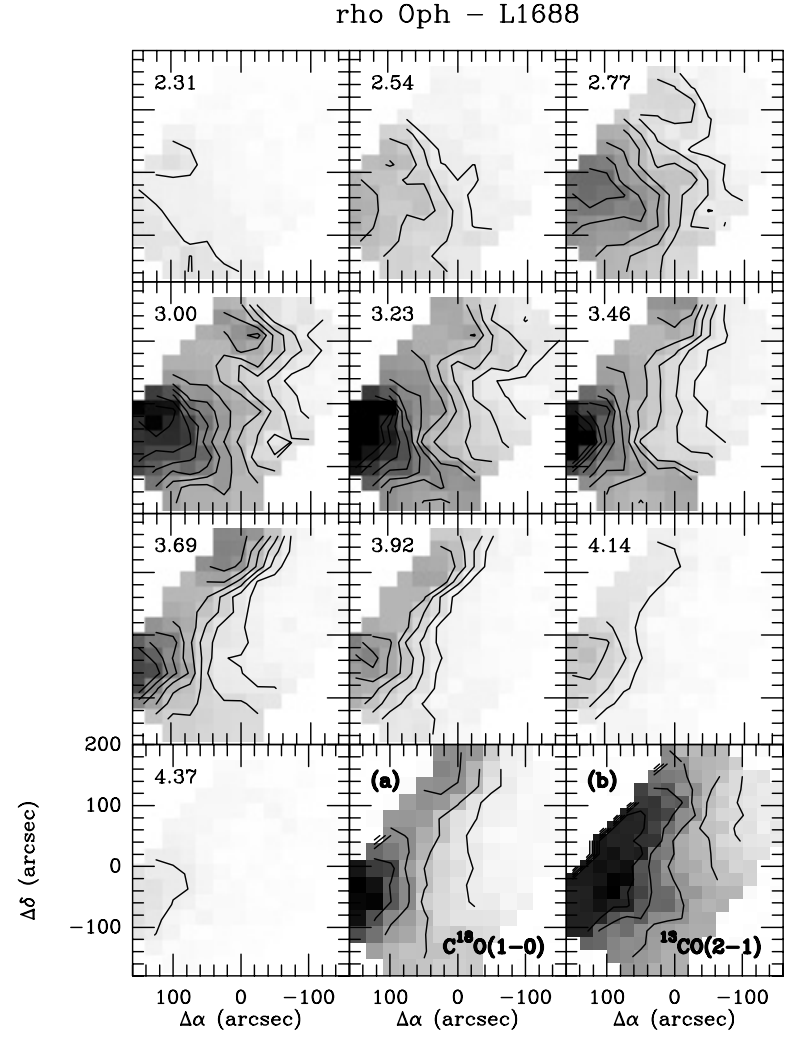

Fig. 6. Channel maps of the $\mathrm{C}^{18} \mathrm{O}(1-0)$ emission $\left(\mathrm{K} \mathrm{km} \mathrm{s}^{-1}, T_{\mathrm{A}}^{*}\right)$ in the L1688-W PDR, as well as integrated intensity maps of $\mathrm{C}^{18} \mathrm{O}(1-0)$ and ${ }^{13} \mathrm{CO}(2-1)\left((\mathbf{a})\right.$ and (b)) in the velocity interval $3.4-4.2 \mathrm{~km} \mathrm{~s}^{-1}$, corresponding to the emission associated to the PDR as seen by ISO. The velocities of each channel map is indicated in the upper left corner. Offsets are given with respect to $\alpha_{2000}=16^{\mathrm{h}} 25^{\mathrm{m}} 58^{\mathrm{s}}$, $\delta_{2000}=-24^{\circ} 21^{\prime} 00^{\prime \prime}$.

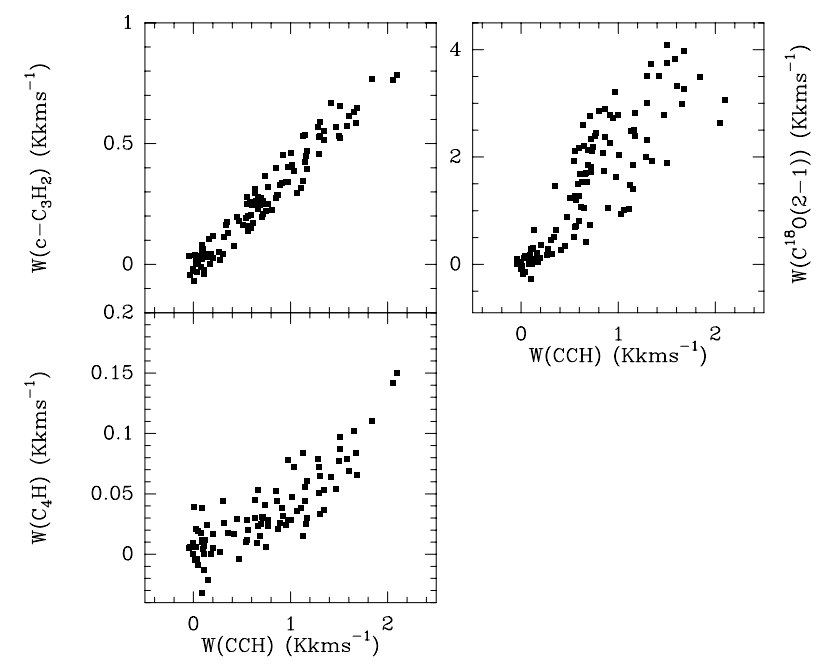

Fig. 7. Scatter diagrams in the Horsehead nebula. The scatter around the zero emission points is indicative of the noise in the data.

cyanopolyynes or density tracers such as CS (see Table 1) and were chosen in order to complete our inventory of the carbon budget. In the Horsehead, these deep integrations were unfortunately performed prior to the large-scale mapping, so that the position observed at the IR peak does actually not 

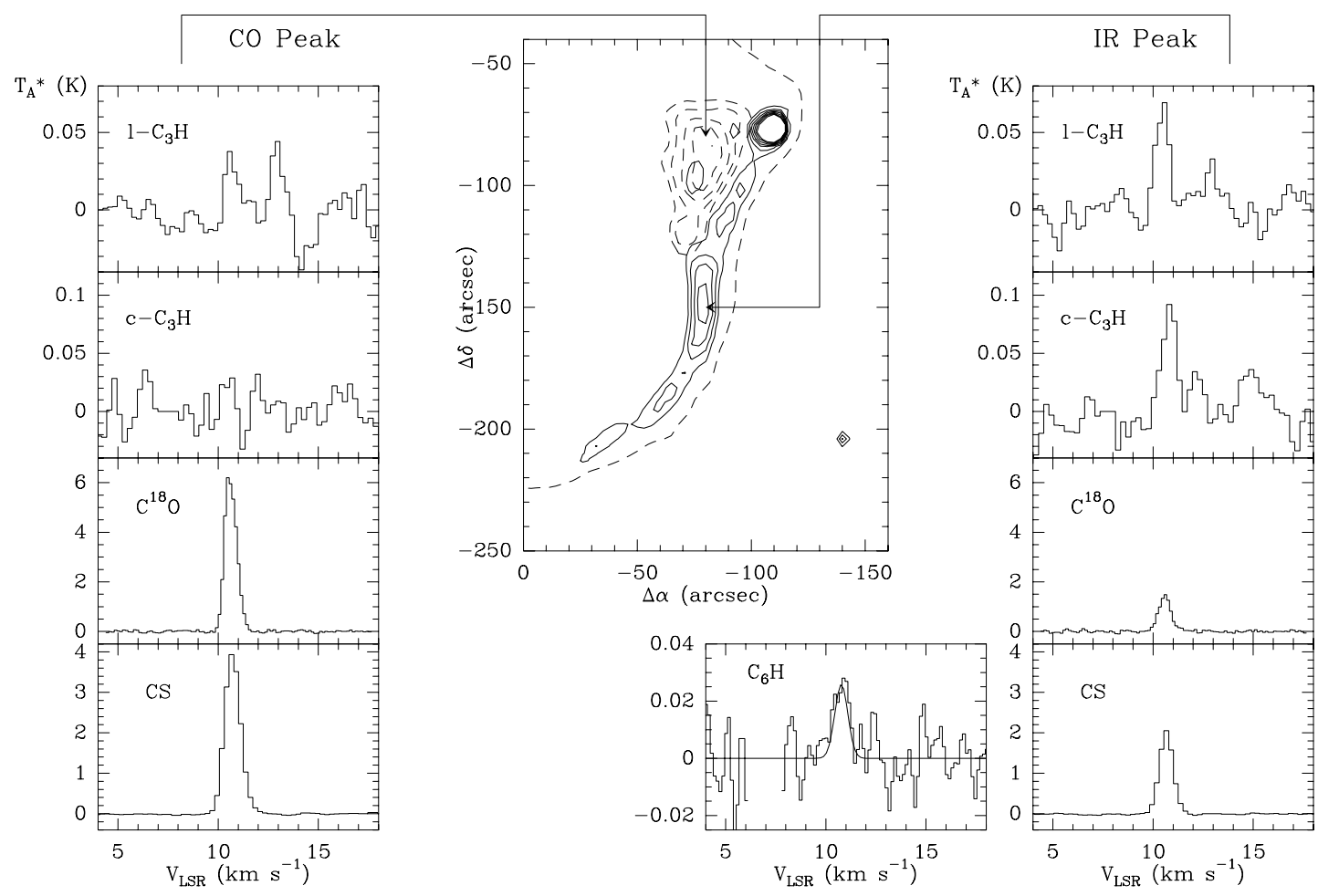

Fig. 8. Sample spectra of heavier hydrocarbons and other species obtained at the so-called CO peak (indicated by dashed ${ }^{12} \mathrm{CO}$ contours, $30 \%$ and 80 to $95 \%$ of the peak, from Abergel et al. 2003) and IR peak (indicated by full line ISO contours 15 to $30 \mathrm{MJy} / \mathrm{sr}$, from Abergel et al. 2002). All temperature are in $\mathrm{K}, T_{\mathrm{A}}^{*}$ scale. The same scales are used for both positions to ease the comparison between the various molecules. The missing channels in the $\mathrm{C}_{6} \mathrm{H}$ spectrum were removed due to spurious features in the backend response. For this line, we also display the Gaussian fit to the line. Offsets are given with respect to $\alpha_{2000}=05^{\mathrm{h}} 40^{\mathrm{m}} 59.0^{\mathrm{s}}, \delta_{2000}=-02^{\circ} 25^{\prime} 31.4^{\prime \prime}$.

correspond to the maximum hydrocarbon emission, as is seen in Fig. 4. The $\mathrm{CO}$ peak position was inferred from the ${ }^{12} \mathrm{CO}$ intensity map observed by Abergel et al. (2003). Although not displayed in Fig. 8, $\mathrm{HN}^{13} \mathrm{C}$ was detected at both positions, and $\mathrm{HC}_{3} \mathrm{~N}$ was observed at the hydrocarbon peak. $l-\mathrm{C}_{3} \mathrm{H}_{2}$, $l-\mathrm{C}_{4} \mathrm{H}_{2}$ and $\mathrm{CH}_{3} \mathrm{C}_{2} \mathrm{H}$ were not detected at either of the positions, while $c-\mathrm{C}_{3} \mathrm{H}$ and $l-\mathrm{C}_{3}$ Hwere detected only at the IR peak. All other molecules were detected to a better than $3 \sigma$ level. We also emphasize the detection of $\mathrm{C}_{6} \mathrm{H}$ at the IR peak which is to our knowledge the first detection of this molecule reported in a PDR. It could however not be observed at the $\mathrm{CO}$ peak.

\subsection{IC 63}

The IC 63 maps are shown in Fig. 5, as well as a cut illustrating the radial profiles of more species across the PDR border (Fig. 10). The correlation observed between the various tracers in the Horsehead is not so striking in the IC 63 maps as this source is barely resolved by the 30 -m observations and the large beam (28" compared to the 6" offered by ISO) significantly smoothes out the details at the PDR border. Smoothing the ISO data to the 30-m resolution however shows a very good agreement between the probed emissions as the two main infrared peaks get smoothed into one single maximum coincident with the molecular emission feature. On the other hand, the $\mathrm{CO}$ and $\mathrm{C}_{2} \mathrm{H}$ peaks coincide fairly well, and are located just North to the south-most aromatic peak at $6.2 \mu \mathrm{m}$ (indicated in Fig. 5). From the observed integrated emission, a beam-deconvolved size of $\sim 40^{\prime \prime} \times 30^{\prime \prime}(F W H M)$ can be inferred for ${ }^{12} \mathrm{CO}$ and $\mathrm{C}_{2} \mathrm{H}$ (the North-East tail is not accounted here).

In IC 63, the deep integrations were done along a cut crossing the south-most AIB emission peak (indicated in Fig. 9), as well as some extra positions inside the cloud tail. $\mathrm{C}_{4} \mathrm{H}$ and $c-\mathrm{C}_{3} \mathrm{H}_{2}$ are in most cases detected, the latter including transitions at 18 and $85 \mathrm{GHz}$ (see Table 1). $\mathrm{C}_{2} \mathrm{H}(3-2)$ is only marginally detected at some positions. The non-detections are similar to the ones reported in the Horsehead nebula, but include as well both isomers of $\mathrm{C}_{3} \mathrm{H}, \mathrm{HN}^{13} \mathrm{C}$ and $\mathrm{C}_{6} \mathrm{H}$.

\section{4. $L 1688-W$}

In L1688-W, the molecular cloud exhibits a complex velocity field, as was already reported by Wilking \& Lada (1983). It is illustrated in Fig. 6 for the area covered in this study. We associate the PDR border as seen by ISO (Abergel et al. 1999) to the molecular emission in the velocities range 3.4 to $4.2 \mathrm{~km} \mathrm{~s}^{-1}$ (see integrated intensity maps in Fig. 6). Along the cut displayed in Fig. 3, the brightest hydrocarbon emission is detected at positions towards the illuminating star where $\mathrm{C}^{18} \mathrm{O}$ has already started to decay significantly (Fig. 11). The emission at negative offsets can nevertheless be due to the particular geometry of L1688-W(Abergel et al. 1999), implying that the PDR is partly seen face-on at probed positions inside the cavity.

In L1688-W, a somewhat smaller number of species was investigated in deeper integrations. Apart from the data presented 


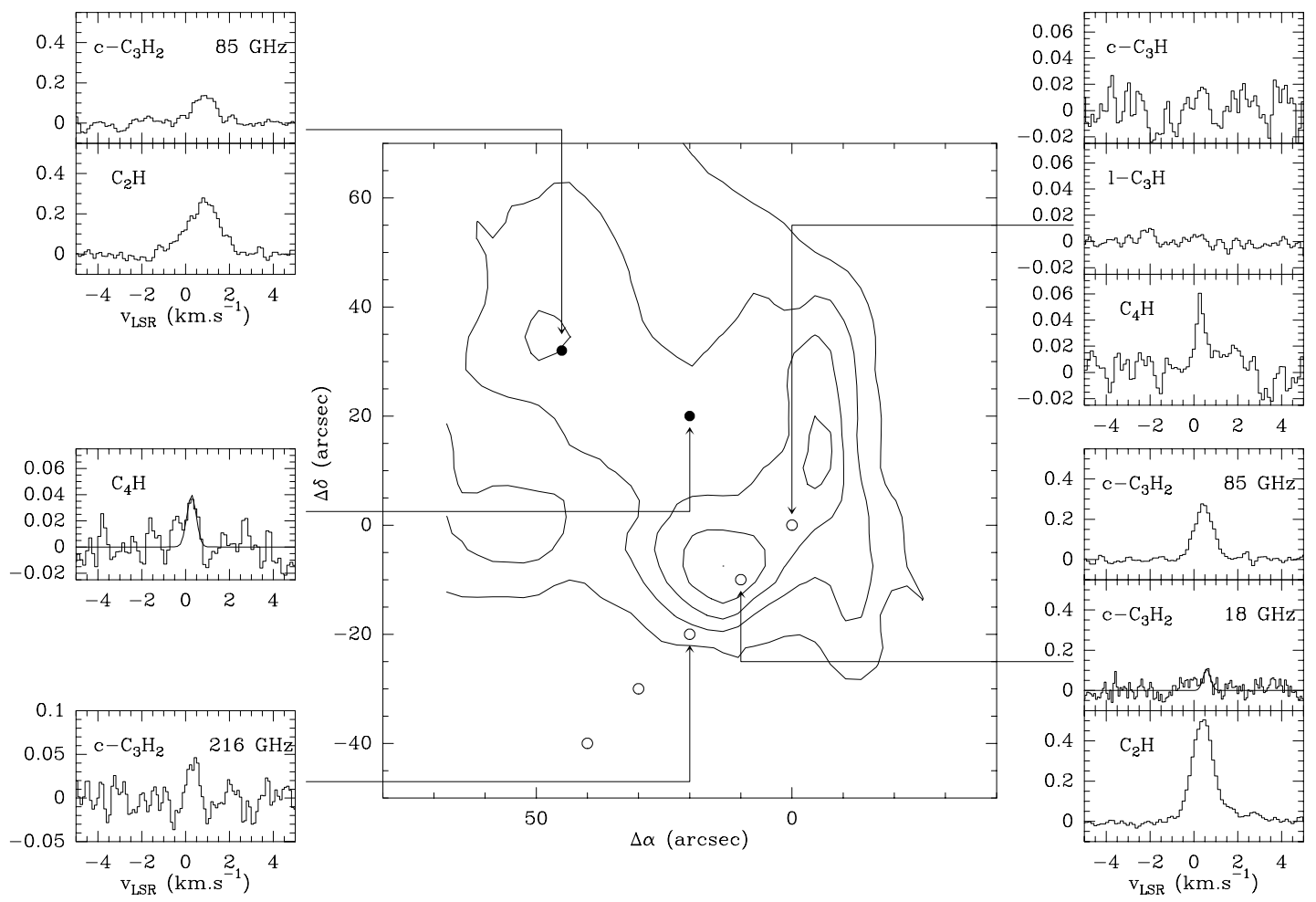

Fig. 9. Sample spectra of weaker hydrocarbons and other species obtained at some dedicated positions in IC 63 displayed over contours of the $6.2 \mu \mathrm{m}$ ISO integrated emission map. It illustrates in particular the non-detection of the cyclic and linear forms of $\mathrm{C}_{3} \mathrm{H}$ around 92 and $98 \mathrm{GHz}$ respectively. The probed positions are marked with filled and open circles. The open circles indicate the cuts displayed in Fig. 10. All temperature are in $\mathrm{K}, T_{\mathrm{A}}^{*}$ scale. Offsets are given with respect to $\alpha_{2000}=00^{\mathrm{h}} 59^{\mathrm{m}} 00.7^{\mathrm{s}}, \delta_{2000}=60^{\circ} 53^{\prime} 19^{\prime \prime}$.

in Fig. 11, observations of $c-\mathrm{C}_{3} \mathrm{H}$ and $l-\mathrm{C}_{3} \mathrm{H}$ were unfruitful down a $5 \mathrm{mK}(1 \sigma)$ noise level.

\section{Analysis of the physico-chemical conditions}

We have used the available collection of species to derive the physical and chemical conditions at work in the three sources, and in particular assess a tentative carbon budget in these PDRs (see Sect. 6.1).

\subsection{Column densities}

As mentioned in the previous section, one should bear in mind that beam dilution effects are expected to affect the measured intensity. This is especially true in our case since we look at positions located close to sharp edges. To first-order, this mainly means that some of the column densities derived below may be under-estimated. The assumptions and methods used to compute the quantities of interest are described in details in Appendix A. We discuss in the following some specific hypothesis on a source to source basis. The results are gathered in Table 2.

\subsubsection{Horshead nebula}

From the ${ }^{12} \mathrm{CO}$ maps of Abergel et al. (2003), we derived $T_{\text {kin }} \sim 40 \pm 2 \mathrm{~K}$ at the PDR location. Using this temperature, the hydrogen number densities deduced from LVG modelling are of the order of some $10^{4} \mathrm{~cm}^{-3}$.

Following the method described in Appendix A.2, we inferred $\mathrm{C}_{2} \mathrm{H}$ excitation temperatures between 5 and $10 \mathrm{~K}$, while $\tau_{\text {tot }}$ never exceeded 1.5. We cross-checked these results using the additional $N=3-2$ transitions observed at some positions. The $N=3-2 / N=1-0$ line intensity ratio are consistent with $T_{\mathrm{ex}}$ in the range $4.5-8 \mathrm{~K}$, in good agreement with the HFS outputs. The results show similar values as the one reported by Fuente et al. $(1993,2003)$ in NGC 7023 and the Orion Bar. The constant density assumed to conduct the $c-\mathrm{C}_{3} \mathrm{H}_{2}$ and $\mathrm{C}_{4} \mathrm{H}$ analysis (see Appendix A.3) was taken to be $2 \times 10^{4} \mathrm{~cm}^{-3}$.

\subsubsection{IC 63}

As discussed previously, the relatively small size of the emitting area compared to the mapping beams requires a correction factor to be applied to the line intensities. We used the approach described by Jansen et al. (1994) to convert $T_{\mathrm{mb}}$ into brightness temperatures within a source filling $1000 \operatorname{arcsec}^{2}$ for ${ }^{12} \mathrm{CO}$ and the hydrocarbon emission, and $400 \operatorname{arcsec}^{2}$ for the ${ }^{13} \mathrm{CO}(2-$ 1) emission. Under these assumptions, the ${ }^{12} \mathrm{CO}$ observations indicate $T_{\text {kin }} \sim 48 \pm 2 \mathrm{~K}$, in excellent agreement with Jansen et al.'s finding of $50 \pm 10 \mathrm{~K}$. Since only one $\mathrm{C}^{18} \mathrm{O}$ transition was available in this source, we made the additional assumption of a volume density $n_{\mathrm{H}_{2}}=5 \pm 2 \times 10^{4} \mathrm{~cm}^{-3}$, based on the results of Jansen et al. (1994). The same density was applied to the 


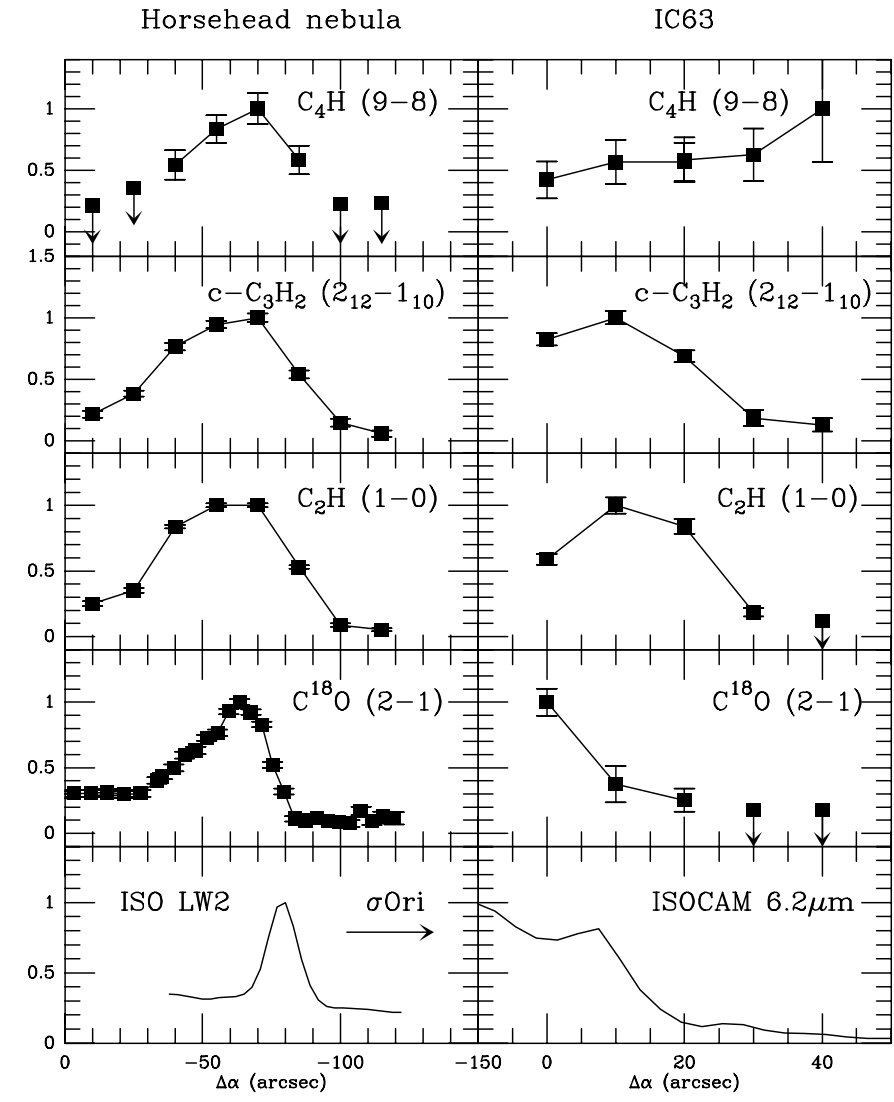

Fig. 10. Normalized line intensities across the PDR fronts of the Horsehead nebula and IC 63. Horsehead: iso-declination cut performed through the IR-peak (see Fig. 4) at $\delta_{2000}=-2^{\circ} 28^{\prime} 00^{\prime \prime}$. All intensities are normalized to the peak (respectively 0.24, 0.9, 2.3 and $8.7 \mathrm{~K}$ from top to bottom, and $24 \mathrm{MJy} / \mathrm{sr}$ for the ISO LW2 profile). The illuminating source lies towards the negative offsets. IC 63: cut performed along the direction indicated by open circles in Fig. 9 . All intensities are normalized to the peak (respectively $0.03,0.27$, 0.5 and $0.2 \mathrm{~K}$ from top to bottom, and $310 \mathrm{MJy} / \mathrm{sr}$ for the ISOCAM CVF profile). The illuminating source lies towards the positive offsets. All error bars are $\pm 1 \sigma$. Upper limits indicate non-detections at a $2 \sigma$ level.

analysis of the $c-\mathrm{C}_{3} \mathrm{H}_{2}$ and $\mathrm{C}_{4} \mathrm{H}$ column densities. The inferred $\mathrm{C}^{18} \mathrm{O}$ column density at $\left(0^{\prime \prime}, 0^{\prime \prime}\right)$ offset is however 3 times lower than the value reported by these authors. This is likely due to the $10^{\prime \prime}$ offset between their position and ours, sufficient to explain such rapid variations in regards of the source size (see Table 2).

The $\mathrm{C}_{2} \mathrm{H}$ analysis indicates that $80 \%$ of the observed points exhibit $\tau_{\text {tot }}<1$ and are consistent with $T_{\mathrm{ex}}=5.8-8 \mathrm{~K}$. The inferred abundances are somewhat higher than the results inferred by Jansen et al. (1994) at a position 10-20" away from ours.

\subsubsection{L1688-W}

The CO kinetic temperatures were derived using an additional $\mathrm{C}^{18} \mathrm{O}(2-1)$ map obtained at the CSO (Gerin, private communication). We calculated $T_{\text {kin }} \sim 21 \pm 2 \mathrm{~K}$ in the region of interest. As already reported by Lada \& Wilking (1980), the CO lines however exhibit significant self-absorption. Despite the dilution effects mentioned above, the temperatures in this source are thus lower limits, implying upper limits for the molecular column densities.

While the $\mathrm{C}_{2} \mathrm{H}$ HFS analysis reveal excitation temperature similar to the ones found in IC 63 , we used the volume density profile derived by Habart et al. (2003) to constrain the $c-\mathrm{C}_{3} \mathrm{H}_{2}$ and $\mathrm{C}_{4} \mathrm{H}$ column densities. The simulations confirm the small optical depth at the probed positions.

\subsection{Fractional abundances}

\subsubsection{Systematics}

The molecular abundances with respect to $\mathrm{H}_{2}$ are compiled in Table 2. Appendix A.6 describes the methods used to derive the $\mathrm{H}_{2}$ column densities in each of the sources. The hydrocarbon abundances appear reasonably constant across the different objects and positions, although to a lesser extent in L1688-W where abundances do not always peak at the intensity maxima. We will discuss this point later. This constancy confirms the general trend already reported in the diffuse gas (Lucas \& Liszt 2000). Although error bars remain high (essentially due to uncertainties on $N\left(\mathrm{H}_{2}\right)$ ), the observed values result in average abundances of $\left[\mathrm{C}_{2} \mathrm{H}\right]=1.9 \pm 0.4 \times 10^{-8},\left[c-\mathrm{C}_{3} \mathrm{H}_{2}\right]=$ $1.4 \pm 0.2 \times 10^{-9}$ and $\left[\mathrm{C}_{4} \mathrm{H}\right]=1.1 \pm 0.2 \times 10^{-9}$.

Abundance ratios between molecules show a somewhat similar trend and seem to be hierarchically distributed. On average, the following ratios are found: $\left[\mathrm{C}^{18} \mathrm{O}\right] /\left[\mathrm{C}_{2} \mathrm{H}\right] \sim 25$ (although with large scatter), $\quad\left[c-\mathrm{C}_{3} \mathrm{H}_{2}\right] /\left[\mathrm{C}_{4} \mathrm{H}\right] \sim 1$ and $\left[\mathrm{C}_{2} \mathrm{H}\right] /\left[c-\mathrm{C}_{3} \mathrm{H}_{2}\right] \sim 15$. The latter result is of the same order as the value reported in the diffuse gas (ratio of $28 \pm 8$ ) by Lucas \& Liszt (2000).

\subsubsection{Horsehead nebula}

In this source, it is interesting to note that the position where the hydrocarbon emission is the largest coincides with a peak of the $\mathrm{H}_{2}$ column density as traced by the continuum emission at $1.2 \mathrm{~mm}$ (Fig. 4). This trend does however not hold for species such as $\mathrm{C}^{18} \mathrm{O}$ (although within large error bars), $\mathrm{CS}$ and $\mathrm{HN}^{13} \mathrm{C}$, whose abundance maxima do not coincide with this $\mathrm{H}_{2}$ column density peak. Note also that the fraction of the beam filled by the cloud is smaller at the hydrocarbon peak than at the $\mathrm{CO}$ peak, thus lowering the intensities measured at the border. This is confirmed by interferometric observations of the PDR border which show an enhancement of the hydrocarbon abundances at the aromatic peak (Pety et al. 2003).

\subsubsection{IC 63}

In IC 63, the small relative variations of abundances observed between the different offsets is very likely related to the proximity of the probed positions with respect to the beam sizes. The $\mathrm{C}^{18} \mathrm{O}$ abundances are significantly lower than the value reported by Jansen et al. (1994). This discrepancy arises from the large $\mathrm{H}_{2}$ column densities assumed at positions probed outwards the cloud. 

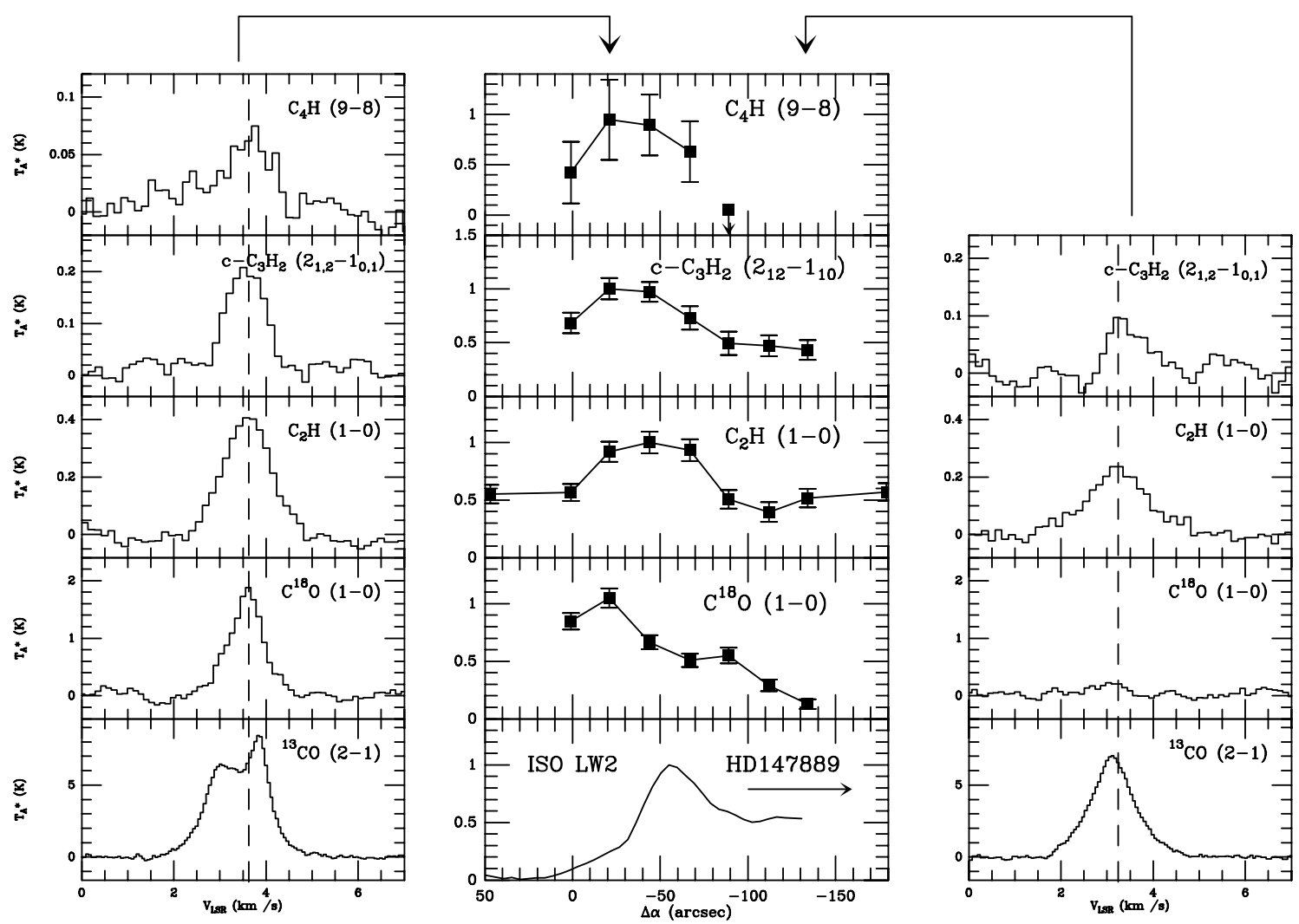

Fig. 11. Normalized line intensities over the cut displayed in Fig. 3 across the L1688-W PDR. Error bars are $\pm 1 \sigma$. The star lies towards the negative offsets. The 0-offset refers to the most North-East point of the cut (Fig. 3). All intensities are normalized to the peak (respectively 0.08, 0.2, 0.43 and $1.6 \mathrm{~K}$ from top to bottom, and $117 \mathrm{MJy} / \mathrm{sr}$ for the ISO LW2 profile). An upper limit is indicated for the rightmost point of $\mathrm{C}_{4} \mathrm{H}$. Sample spectra at two offsets are also shown. The dashed line indicates the centroid velocity of the optically thin lines, illustrating the self-absorption in ${ }^{13} \mathrm{CO}$. positive offsets). All temperature are in $\mathrm{K}, T_{\mathrm{A}}^{*}$ scale.

\subsubsection{L1688-W}

Firm conclusions are more difficult to draw in L1688-W as the available sample remains restricted to few points. If our picture of the variations of the $\mathrm{H}_{2}$ column density is correct, the highest hydrocarbon abundances are not found at their corresponding intensity peaks but closer to the illuminating star (Fig. 11). Again, this can be due to the particular geometry of the PDR partly seen face-on. Still, the $\mathrm{C}^{18} \mathrm{O}$ abundances experience a drop much steeper than the hydrocarbons in the direction of the illuminating star.

\section{Carbon chemistry}

\subsection{Carbon budget}

The compilation of molecular abundances allows us to derive a tentative carbon budget representative of PDR conditions. For comparison, Table 3 also includes results obtained in the diffuse ISM (Lucas \& Liszt 2000), as well as in a typical carbonrich dark cloud (see also Sect. 6.2). For this, we have co-added typical abundances for molecules with a given number of carbon atoms. Since all molecules are not observed in the same sources, this carbon budget has only a statistical meaning, and numbers cannot be very accurate. The contribution of carbon clusters containing up to $2-3$ atoms is very similar for all three media, with a clear abundance decrease as the molecular size increases, both in PDRs and dark clouds. Heavier chains and rings (4 carbon atoms and more) are however less abundant in PDRs and diffuse clouds than in dark clouds by a factor of order 5-10. The fraction of carbon tied to more complex molecules may be only partly representative of the real situation. A lower number of molecules of that size is detected and other species still remain to be identified.

\subsection{Comparison to dark clouds}

We have compared the carbon chain and ring abundances to results obtained in TMC-1 (e.g. Pratap et al. 1997) and L134N (L183, e.g. Dickens et al. 2000), two dark clouds known as efficient carbon factories. The carbon budget corresponding to the dark cloud conditions is also indicated in Table 3. As for the PDRs, it remains difficult to derive accurate estimates of the total $\mathrm{H}_{2}$ column densities in these objects. These estimates are indeed usually affected by strong molecular depletion (e.g. Cernicharo et al. 1987; Alves et al. 1999) and possible evolution of the grain properties in the cold and dense medium characteristic of dark clouds (e.g. Stepnik et al. 2003; Kramer et al. 2003). The abundances reported in Table 3 have been calculated assuming an averaged extinction of order 20 mag, which may lie in between the actual values applying to TMC-1 and L134N. In addition we have decided to compare the fractional abundances with respect to $\mathrm{C}_{2} \mathrm{H}$, the most 
Table 2. Column densities and abundances relative to $\mathrm{H}_{2}$ (boldface) inferred at positions of interest in the Horsehead, IC 63 and L1688-W. (d) $10^{12} \mathrm{~cm}^{-2}$. Units for column densities are in $\mathrm{cm}^{-2}$ and indicated in superscript for each molecule. Units for abundances are indicated in the first column of each corresponding row. The offsets refer to the positions indicated Figs. 6 and 9.

\begin{tabular}{|c|c|c|c|c|c|c|c|c|}
\hline \multirow[t]{2}{*}{ Molecule } & \multicolumn{3}{|c|}{ Horsehead nebula } & \multicolumn{3}{|c|}{ IC 63 cut } & \multicolumn{2}{|c|}{ L1688-W cut } \\
\hline & $\mathrm{CO}$ peak & IR peak & Hydroc. peak & $\left(0^{\prime \prime}, 0^{\prime \prime}\right)$ & $\left(10^{\prime \prime},-10^{\prime \prime}\right)$ & $\left(20^{\prime \prime},-20^{\prime \prime}\right)$ & $\left(\Delta \alpha=-21^{\prime \prime}\right)$ & $\left(\Delta \alpha=-134^{\prime \prime}\right)$ \\
\hline $\mathrm{H}_{2}{ }^{\left(10^{21}\right)}$ & $7.5 \pm 2.5$ & $7.2 \pm 2.4$ & $10.5 \pm 3.5$ & $5.0 \pm 2.0$ & $5.0 \pm 2.0$ & $5.0 \pm 2.0$ & $11.4 \pm 2.4$ & $2.4 \pm 0.3$ \\
\hline $\mathrm{C}^{18} \mathrm{O}^{\left(10^{15}\right)}$ & $5.0 \pm 0.2$ & $3.5 \pm 0.2$ & $4.0 \pm 0.2$ & $0.18 \pm 0.02$ & $0.05 \pm 0.02$ & $0.05 \pm 0.02$ & $4.0 \pm 0.2$ & $0.4 \pm 0.02$ \\
\hline$\left(10^{-7}\right)$ & $6.7 \pm 2.2$ & $4.9 \pm 1.6$ & $3.8 \pm 1.3$ & $0.36 \pm 0.15$ & $0.10 \pm 0.06$ & $0.10 \pm 0.06$ & $8.0 \pm 3.2$ & $1.7 \pm 0.2$ \\
\hline $\mathrm{C}_{2} \mathrm{H}^{\left(10^{13}\right)}$ & $17.0 \pm 2.0$ & $16.0 \pm 2.0$ & $22.7 \pm 3.3$ & $5.3 \pm 0.4$ & $8.6 \pm 0.6$ & $6.9 \pm 0.5$ & $3.0 \pm 0.3$ & $2.0 \pm 0.2$ \\
\hline$\left(10^{-8}\right)$ & $2.3 \pm 0.8$ & $2.2 \pm 0.8$ & $2.2 \pm 0.8$ & $1.1 \pm 0.4$ & $2.1 \pm 1.0$ & $1.4 \pm 0.6$ & $0.6 \pm 0.2$ & $0.8 \pm 0.1$ \\
\hline$c-\mathrm{C}_{3} \mathrm{H}^{\left(10^{12}\right)}$ & $<2.1$ & $3.9 \pm 0.5$ & - & $<1.2$ & - & - & - & - \\
\hline$\left(10^{-10}\right)$ & $<2.8$ & $5.4 \pm 1.9$ & - & $<2.4$ & - & - & - & - \\
\hline$l-\mathrm{C}_{3} \mathrm{H}^{\left(10^{12}\right)}$ & $<2.3$ & $2.1 \pm 0.7$ & - & $<5.8$ & - & - & - & - \\
\hline$\left(10^{-10}\right)$ & $<3.1$ & $2.9 \pm 1.4$ & - & $<11.6$ & - & - & - & - \\
\hline$c-\mathrm{C}_{3} \mathrm{H}_{2}{ }^{\left(10^{12}\right)}$ & $11.0 \pm 3.0$ & $9.3 \pm 0.2$ & $15.0 \pm 5.0$ & $4.5 \pm 1.0$ & $4.7 \pm 1.0$ & $3.0 \pm 0.6$ & $3.0 \pm 0.6$ & $3.0 \pm 0.6$ \\
\hline$\left(10^{-9}\right)$ & $1.5 \pm 0.6$ & $1.3 \pm 4.3$ & $1.4 \pm 0.7$ & $0.9 \pm 0.4$ & $0.9 \pm 0.4$ & $0.6 \pm 0.3$ & $0.6 \pm 0.3$ & $1.3 \pm 0.3$ \\
\hline$l-\mathrm{C}_{3} \mathrm{H}_{2}\left({ }^{10^{12}}\right)$ & $<0.32$ & $<0.33$ & - & - & - & - & - & - \\
\hline$\left(10^{-11}\right)$ & $<4.3$ & $<4.6$ & - & - & - & - & - & - \\
\hline $\mathrm{CH}_{3} \mathrm{C}_{2} \mathrm{H}^{\left(10^{13}\right)}$ & $<1.1$ & $<1.1$ & - & - & - & - & - & - \\
\hline$\left(10^{-9}\right)$ & $<\mathbf{1 . 4}$ & $<\mathbf{1 . 4}$ & - & - & - & - & - & - \\
\hline $\mathrm{C}_{4} \mathrm{H}^{\left(10^{12}\right)}$ & $10.0 \pm 3.0$ & $11.0 \pm 3.0$ & $31.0 \pm 7.0$ & $3.3 \pm 0.2$ & $3.7 \pm 0.4$ & $6.5 \pm 1.5$ & $15.0 \pm 3.0$ & - \\
\hline$\left(10^{-9}\right)$ & $1.3 \pm 0.6$ & $1.5 \pm 0.7$ & $3.0 \pm 1.2$ & $0.6 \pm 0.3$ & $0.7 \pm 0.3$ & $1.3 \pm 0.6$ & $3.0 \pm 1.3$ & - \\
\hline $\mathrm{C}_{6} \mathrm{H}^{\left(10^{12}\right)}$ & - & - & $1.0 \pm 0.5$ & - & - & - & - & - \\
\hline$\left(10^{-10}\right)$ & - & - & $1.0 \pm 0.6$ & - & - & - & - & - \\
\hline $\mathrm{HN}^{13} \mathrm{C}^{\left(10^{12}\right)}$ & $1.2 \pm 0.2$ & $0.3 \pm 0.1$ & - & $<0.11$ & - & - & - & - \\
\hline$\left(10^{-10}\right)$ & $1.6 \pm 0.6$ & $0.4 \pm 0.2$ & - & $<\mathbf{0 . 2}$ & - & - & - & - \\
\hline $\mathrm{HC}_{3} \mathrm{~N}^{\left(10^{12}\right)}$ & - & - & $0.6 \pm 0.2$ & - & - & - & - & - \\
\hline$\left(10^{-11}\right)$ & - & - & $5.7 \pm 2.7$ & - & - & - & - & - \\
\hline $\mathrm{CS}^{\left(10^{13}\right)}$ & $12 \pm 2$ & $2.9 \pm 0.5$ & - & $0.6 \pm 0.1$ & $0.4 \pm 0.1$ & $0.15 \pm 0.03$ & - & - \\
\hline$\left(10^{-8}\right)$ & $1.6 \pm 0.6$ & $4.0 \pm 1.5$ & - & $0.12 \pm 0.05$ & $0.08 \pm 0.04$ & $\mathbf{0 . 0 3} \pm \mathbf{0 . 0 1}$ & - & - \\
\hline
\end{tabular}

abundant hydrocarbon in our sample. Assuming again an extinction of $20 \mathrm{mag}$, the $\mathrm{C}_{2} \mathrm{H}$ fractional abundances to $\mathrm{H}_{2}$ at the TMC- 1 cyanopolyyne peak $(\mathrm{CP})$ and at the $\mathrm{L} 134 \mathrm{~N}$ hydrocarbon peak would however be $4 \times 10^{-9}$ and $8 \times 10^{-9}$ respectively, somewhat lower than the values we have derived in PDRs. This is also true for species such as $c-\mathrm{C}_{3} \mathrm{H}_{2}$ or $\mathrm{C}_{4} \mathrm{H}$.

Table 4 compiles the abundances relative to $\mathrm{C}_{2} \mathrm{H}$ for each of the sources at the positions of interest. The error bars have not been considered as we look here at general trends. Contrary to the abundances relative to $\mathrm{H}_{2}$, the fractional abundances relative to $\mathrm{C}_{2} \mathrm{H}$ are generally larger in the dark clouds than in our sample PDRs (we will here not address the differences between TMC-1 and L134N, see Fossé 2003). For all molecules but $l-\mathrm{C}_{3} \mathrm{H}$, there is at least a factor of 5 difference between the molecular fractions in the PDRs and in TMC-1. This is particularly true for abundant species such as $c-\mathrm{C}_{3} \mathrm{H}_{2}$ and $\mathrm{C}_{4} \mathrm{H}$, whereas they seem to have similar contributions to the carbon budget in both media (Table 3 ). This behaviour is very likely due to the different chemistries at work in the two media, and will be further discussed in the next section. We also note the enormous drop of $\left[\mathrm{HC}_{3} \mathrm{~N}\right] /\left[\mathrm{C}_{2} \mathrm{H}\right]$ between TMC-1 $\mathrm{CP}$ and the Horsehead nebula (factor of 1000). Although we probably missed the "cyanopolyyne" peak position of the Horsehead, the difference is still meaningful.

\subsection{Comparison to models}

In this section, we compare our observed data with model predictions. We used a plane parallel PDR model which solves self-consistently the thermal and chemical balance of a semiinfinite homogeneous slab (Le Bourlot et al. 1993; Le Petit et al. 2002). We describe the results for physical conditions mimicking those of the Horsehead nebula and the $\rho$ Oph interface, namely a gaz density of $n_{\mathrm{H}}=2 \times 10^{4} \mathrm{~cm}^{-3}$ and a radiation field multiplied by $G_{0}=100$ relative to the ISRF determined by Habing (1968). Since the chemical networks have not been fully tested for large molecules, we have chosen to use two different networks, the New Standard Model developed by Herbst and collaborators (Terzieva \& Herbst 1998) and the UMIST95 chemical rate file (Millar et al. 1997). Both chemical networks 
Table 3. Carbon budget derived in PDRs (this work), diffuse ISM (from Bell et al. 1983; Federman et al. 1994; Shuping et al. 1999; Lucas \& Liszt 2000; Roueff et al. 2002; Cernicharo et al. 2002; Rachford et al. 2002) and dark clouds (from Pratap et al. 1997; Bell et al. 1998; Dickens et al. 2000; Turner et al. 2000; Fossé et al. 2001; Fossé 2003). Note that, for a given number of carbon atoms, the molecules found in the different regions may differ (e.g. no $\mathrm{C}_{n}$ carbon clusters in PDRs and dark clouds). For the diffuse ISM, we used the $\mathrm{C} / \mathrm{H}$ abundance ratio of $1.4 \times 10^{-4}$ reported by Cardelli et al. (1996) and assumed an $\mathrm{H} / \mathrm{H}_{2}$ abundance ratio of 0.6 as observed in the $\zeta$ Oph line of sight (e.g. Rachford et al. 2002).

\begin{tabular}{lccc}
\hline \hline Number of carbon atoms & & $N_{\text {Tot }} / N\left(\mathrm{H}_{2}\right)$ \\
& PDRs & Diffuse ISM & Dark clouds \\
\hline $1\left(\mathrm{C}^{+}, \mathrm{C}, \mathrm{CO}\right)$ & $2.6 \times 10^{-4}$ & $2.3 \times 10^{-4}$ & $8.0 \times 10^{-5}$ \\
$1\left(\mathrm{CH}^{+}, \mathrm{CH}, \mathrm{CS}, \mathrm{HCN}, ..\right)$ & $7.0 \times 10^{-8}$ & $5.0 \times 10^{-8}$ & $\leq 1.0 \times 10^{-7}$ \\
$2\left(\mathrm{C}_{2}, \mathrm{C}_{2} \mathrm{H}, \mathrm{C}_{2} \mathrm{~S}, ..\right)$ & $8.0 \times 10^{-8}$ & $8.0 \times 10^{-8}$ & $1.0 \times 10^{-8}$ \\
$3\left(\mathrm{C}_{3}, \mathrm{C}_{3} \mathrm{H}, \mathrm{C}_{3} \mathrm{H}_{2}, \mathrm{HC}_{3} \mathrm{~N}_{,} \mathrm{CH}_{3} \mathrm{C}_{2} \mathrm{H}, ..\right)$ & $5.0 \times 10^{-9}$ & $4.5 \times 10^{-9}$ & $1.2 \times 10^{-8}$ \\
$4\left(\mathrm{C}_{4}, \mathrm{C}_{4} \mathrm{H}, \mathrm{C}_{4} \mathrm{H}_{2}, ..\right)$ & $2.0 \times 10^{-9}$ & $1.5 \times 10^{-9}$ & $1.7 \times 10^{-8}$ \\
$5\left(\mathrm{C}_{5}, \mathrm{C}_{5} \mathrm{H}, \mathrm{HC}_{5} \mathrm{~N}, ..\right)$ & - & - & $1.2 \times 10^{-9}$ \\
$6\left(\mathrm{C}_{6} \mathrm{H}, \mathrm{C}_{6} \mathrm{H}_{2}, ..\right)$ & $1.1 \times 10^{-10}$ & - & $4.2 \times 10^{-10}$ \\
$7\left(\mathrm{HC}_{7} \mathrm{~N}, ..\right)$ & - & - & $5.5 \times 10^{-10}$ \\
$8\left(\mathrm{C}_{8} \mathrm{H}, ..\right)$ & - & - & $1.1 \times 10^{-11}$ \\
\hline
\end{tabular}

Table 4. Fractional abundances relative to $\mathrm{C}_{2} \mathrm{H}$. The assumed column densities of $\mathrm{C}_{2} \mathrm{H}$ are recalled in the first row. $\mathrm{CP}$ stands for Cyanopolyyne Peak. References are: ${ }^{(a)}$ Pratap et al. 1997, ${ }^{(b)}$ Fossé et al. 2001, ${ }^{(c)}$ Guélin et al. 1982, ${ }^{(d)}$ Fossé 2003, ${ }^{(e)}$ Dickens et al. $2000,{ }^{(f)}$ Takano et al. 1998. Since no $\mathrm{C}_{6} \mathrm{H}$ and $\mathrm{HC}_{3} \mathrm{~N}$ measurements were available at the Horsehead IR peak, the corresponding abundances relative to $\mathrm{C}_{2} \mathrm{H}$ are given for the hydrocarbon peak.

\begin{tabular}{lccccc}
\hline \hline Molecule & $\begin{array}{c}\text { Horsehead } \\
\text { IR peak area }\end{array}$ & $\begin{array}{c}\text { IC 63 } \\
\left(0^{\prime \prime},-0^{\prime \prime}\right)\end{array}$ & $\begin{array}{c}\text { L1688-W } \\
\left(\Delta \alpha=-21^{\prime \prime}\right)\end{array}$ & $\begin{array}{c}\text { TMC-1 } \\
(\mathrm{CP})\end{array}$ & L134N \\
\hline $\mathrm{C}_{2} \mathrm{H}\left(10^{13} \mathrm{~cm}^{-2}\right)$ & 16 & 5.3 & 3.0 & $7.2^{(a)}$ & $15^{(d)}$ \\
\hline$c-\mathrm{C}_{3} \mathrm{H}$ & 0.024 & $<0.023$ & - & $0.140^{(b)}$ & $0.050^{(d)}$ \\
$l-\mathrm{C}_{3} \mathrm{H}$ & 0.013 & $<0.110$ & - & $0.012^{(b)}$ & $0.040^{(d)}$ \\
$c-\mathrm{C}_{3} \mathrm{H}_{2}$ & 0.058 & 0.085 & 0.100 & $0.800^{(b)}$ & $0.330^{(d)}$ \\
$l-\mathrm{C}_{3} \mathrm{H}_{2}$ & $<0.002$ & - & - & $0.030^{(b)}$ & $0.004^{(d)}$ \\
$\mathrm{CH}_{3} \mathrm{C}_{2} \mathrm{H}$ & $<0.069$ & - & - & $1.4^{(a)}$ & $0.070^{(d)}$ \\
$\mathrm{C}_{4} \mathrm{H}$ & 0.069 & 0.062 & 0.500 & $2.8^{(c)}$ & $0.735^{(d)}$ \\
$\mathrm{C}_{6} \mathrm{H}$ & 0.004 & - & - & $0.115^{(b)}$ & $0.008^{(d)}$ \\
$\mathrm{HN}{ }^{13} \mathrm{C}$ & 0.002 & $<0.002$ & - & $0.056^{(a)}$ & $0.054^{(e)}$ \\
$\mathrm{HC}_{3} \mathrm{~N}$ & 0.003 & - & - & $2.2^{(f)}$ & $0.127^{(e)}$ \\
\hline
\end{tabular}

include about 400 hundred species with up to 8 carbon atoms, and 4000 chemical reactions. The various possible isomers are not differenciated in the chemical networks.

The photodissociation of $\mathrm{H}_{2}$ and $\mathrm{CO}$ and the photoionization of $\mathrm{C}$ are treated specifically in the PDR code via the computation of the actual ultraviolet radiative transfer in the presence of the complex chemistry provided by the two chemical networks. Then, the transition of atomic to molecular hydrogen is identical in both models. However, the balance between neutral carbon and carbon containing species in the intermediate range of $A_{\mathrm{V}}$ is dependent both on the photo-processes (identical in the two networks) and the individual chemical reactions which involve different assumptions.

Except for the formation of molecular hydrogen, we have not considered any reaction on grain surfaces. All molecules are therefore formed and destroyed in the gas phase. For the external layers of the cloud where the radiation field is the strongest, the main destruction process is photo-dissociation, while chemical reactions are more important in well shielded regions. PAHs are taken into account for their role in the gas heating, but do not participate in the chemistry. Using two networks helps us in setting error bars on model predictions and therefore in identifying agreement or discrepancy between our observed data and the model predictions more securely.

The PDR model assumes steady state equilibrium for both the thermal processes and the chemistry. Given the large density and radiation field, this assumption should be justified since the chemical time scales are dominated by the photodissociation reactions which are very rapid in the conditions we modelled. Furthermore, there is no need to invoke time dependent chemistry for the synthesis of molecules since ionised and atomic carbon are present with a large abundance in the external layers (up to $A_{\mathrm{V}}=4-5 \mathrm{mag}$ for $\mathrm{C}$ ), and can participate in a large variety of chemical reactions for building molecules. 

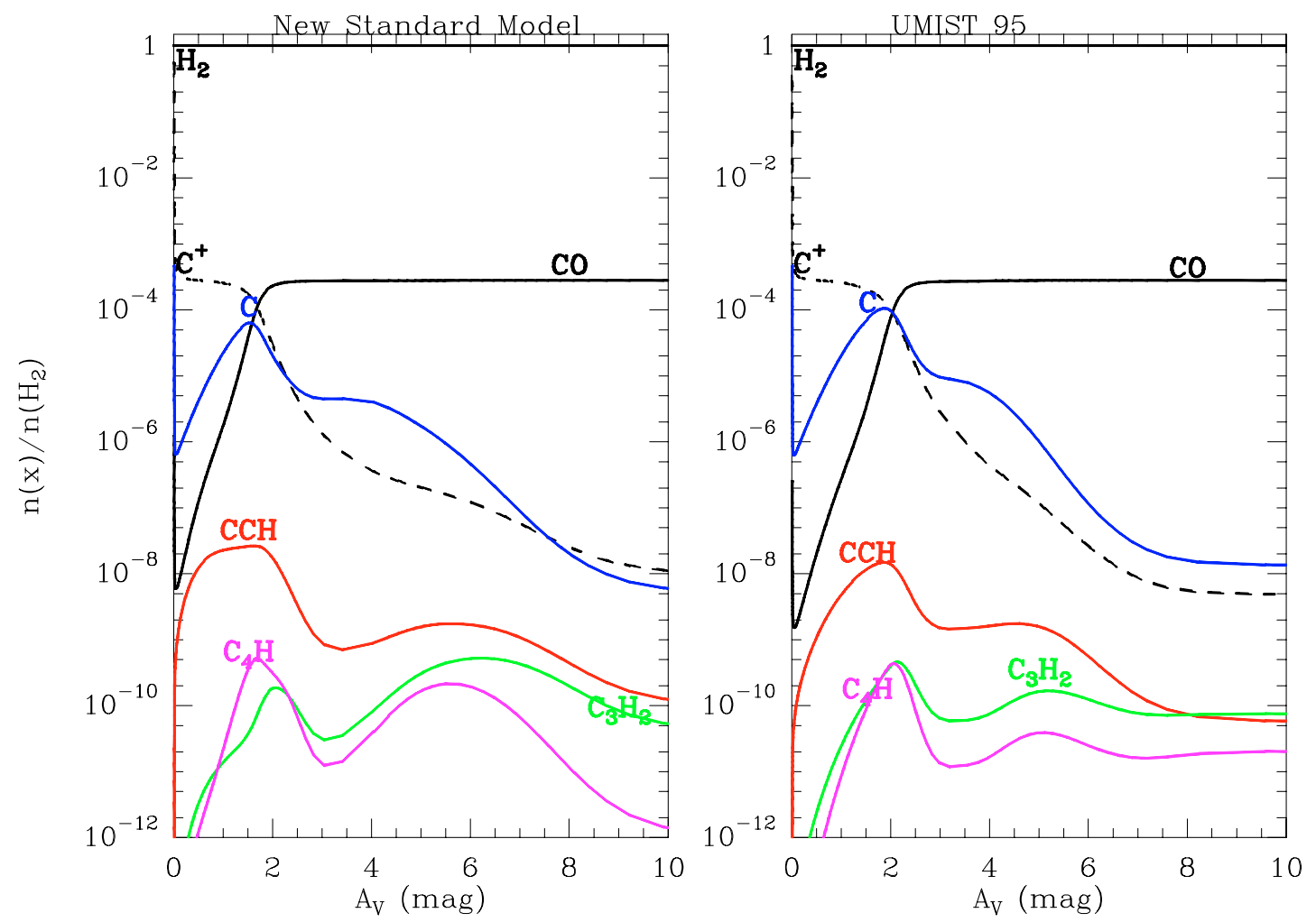

Fig. 12. Prediction for the abundances of various species derived from an updated Le Bourlot et al.'s PDR model, using two chemistry networks, namely Herbst et al.'s New Standard Model (left) and UMIST95 of Millar and collaborators (right). We assumed a gas density of $n_{\mathrm{H}}=$ $2 \times 10^{4} \mathrm{~cm}^{-3}$ and a radiation field $G_{0}=100$. Both models predict too low abundances for $c-\mathrm{C}_{3} \mathrm{H}_{2}$ and $\mathrm{C}_{4} \mathrm{H}$, but also that $\mathrm{C}_{2} \mathrm{H}$ is present in the UV illuminated gas as observed.

We show in Fig. 12 model predictions for the two chemical networks separately. Though the transition from atomic to molecular hydrogen is very similar for both chemical networks, the transition from ionised carbon to neutral carbon, then to carbon monoxide, shows small differences, with the formation of carbon monoxide occurring at slightly smaller $A_{\mathrm{V}}$ in the NSM compared to UMIST95.

We now discuss the predictions for $\mathrm{C}_{2} \mathrm{H}, \mathrm{C}_{3} \mathrm{H}_{2}$ and $\mathrm{C}_{4} \mathrm{H}$. For both models, the abundance of hydrocarbons presents two peaks, the first one located near $A_{\mathrm{V}}=2$, and a second one near $A_{\mathrm{V}}=5$. We shall compare our observed data with peak values in the first peak, which presents the largest abundances. Given the low spatial resolution of the data in comparison with the expected spatial variations, the observed abundances are lower limits to the true abundances. For the Horsehead nebula we have good evidence from the maps that emission lines of $\mathrm{C}_{2} \mathrm{H}$ and related molecules peak in the same region as the mid-IR emission and that the present data could not resolve this sharp peak.

In both models, the peak abundances relative to $\mathrm{H}_{2}$ are $\left[\mathrm{C}_{2} \mathrm{H}\right] \simeq 2 \times 10^{-8},\left[\mathrm{C}_{3} \mathrm{H}_{2}\right] \simeq 2 \times 10^{-10}$ and $\left[\mathrm{C}_{4} \mathrm{H}\right] \simeq 2 \times 10^{-10}$. The peak CO abundance is $\simeq 2.6 \times 10^{-4}$, since all gas phase carbon is locked into $\mathrm{CO}$ for $A_{\mathrm{V}}$ larger than 2 mag. Compared to the observed data, models predict similar abundances relative to $\mathrm{H}_{2}$ for $\mathrm{C}_{2} \mathrm{H}$, and $\mathrm{CO}$; also $\mathrm{C}_{4} \mathrm{H}$ and $\mathrm{C}_{3} \mathrm{H}_{2}$ behave in the same way as we observed. However, their predicted abundances relative to $\mathrm{H}_{2}$ are lower by an order of magnitude than abundances derived from the observations. The discrepancy is clear in both models, all the more if we consider that the peak abundance is obtained over a fairly narrow range of $A_{\mathrm{V}}$. Since our data are taken with a low spatial resolution, we suffer from beam dilution as explained above. How can we explain this discrepancy? We suggest three possibilities which are not independent:

- The photo-dissociation rates for hydrocarbons (molecules, radicals and ions) included in the chemical networks may be overestimated by one order of magnitude. For most species, the values used in models are indeed best guesses with large uncertainties (see van Dishoeck 1988; Fossé 2003).

- Although including thousands of reactions, chemical networks used for models may not list the relevant chemical reactions for forming carbon chains and radicals yet. A related possibility is that some reaction rates are incorrect. For example the role of atomic oxygen in destroying carbon chains and cycles in the interstellar medium is still not settled (Herbst, priv. com.). There is also the possibility that neutral neutral reactions involving neutral carbon, $\mathrm{CH}, \mathrm{C} 2 \mathrm{H}$ and small hydrocarbons may be at work and contribute to the formation of the larger hydrocarbons. Reactions of atomic carbon with acetylene, ethylene, propyne and allene have indeed been studied by Chastaing et al. (2001) and shown to be rapid.

- Another mechanism for producing carbon chains is needed, in addition to gas phase chemistry. An attractive possibility is the erosion and/or destruction of PAHs and small 
carbon grains. Indeed the main process constraining the smallest possible size of PAHs is the destruction of their carbon skeleton. Various mechanisms have been proposed (see Allain et al. 1996; Scott et al. 1997; Le Page et al. 2001, 2003; Verstraete et al. 2001) which all lead to the production of small carbon fragments $\left(\mathrm{C}_{2} \mathrm{H}_{2}, \mathrm{C}_{2}\right.$ or $\left.\mathrm{C}_{3}, \ldots\right)$. Once released in the gas phase, these fragments may further react and take part in the synthesis of the carbon chains and radicals we observed. Given the large fraction of total carbon in solid form (PAHs can represent up to $10 \%$ of total C) compared with the rather small abundance of carbon chains (see Table 3), the production of carbon clusters and molecules from the PAHs is a plausible mechanism. Such a mechanism was also mentioned by Fuente et al. (2003) to explain the high hydrocarbon abundances observed in NGC 7023 and the Orion Bar.

\section{Conclusions}

We have conducted an extensive search for small hydrocarbon chains and rings in three Photon-Dominated Regions. The map obtained in the millimetre transitions of $\mathrm{C}_{2} \mathrm{H}, c-\mathrm{C}_{3} \mathrm{H}_{2}$ and $\mathrm{C}_{4} \mathrm{H}$ show that all three hydrocarbons are found until the border of the PDRs, despite the strong radiation fields $\left(G_{0} \sim 100-1000\right)$. They moreover correlate well with each other, in agreement with trends reported in diffuse clouds (Lucas \& Liszt 2000). In the Horsehead nebula, the hydrocarbon large-scale distribution seems closely related to the emission of aromatic bands observed in the mid-IR, a result in contrast with the $\mathrm{CO}$ brightness distribution. A firm link between the small hydrocarbons and the aromatic band carriers however still requires highresolution maps of this area (see Pety et al. 2003). Heavier species have been probed at positions of interest. They are mostly detected in the Horsehead nebula, with a noticeable detection of $\mathrm{C}_{6} \mathrm{H}$ around $81.8 \mathrm{GHz}$. To our knowledge, this is the first detection of this heavy carbon chain reported in a PDR.

We have derived the hydrocarbon column densities and abundances relative to $\mathrm{H}_{2}$ and $\mathrm{C}_{2} \mathrm{H}$. On the average, the column densities of $\mathrm{C}^{18} \mathrm{O}$ and of the most abundant hydrocarbons are similar in all three PDRs, and comparable to results reported in other sources (e.g. Fuente et al. 1993, 2003). They exhibit representative ratios of order $\sim 25,1$ and 15 for $\left[\mathrm{C}^{18} \mathrm{O}\right] /\left[\mathrm{C}_{2} \mathrm{H}\right]$, $\left[c-\mathrm{C}_{3} \mathrm{H}_{2}\right] /\left[\mathrm{C}_{4} \mathrm{H}\right]$ and $\left[\mathrm{C}_{2} \mathrm{H}\right] /\left[c-\mathrm{C}_{3} \mathrm{H}_{2}\right]$, respectively. We have compared these values to column densities reported in the TMC-1 and L134N dark clouds, known as efficient carbon factories. Although $\mathrm{H}_{2}$ column densities may be underestimated in the dark clouds, abundances relative to $\mathrm{H}_{2}$ are similar up to chains and rings of 2-3 carbon atoms. The same trend is evidenced in carbon budgets derived for PDRs, diffuse clouds and dark clouds conditions. Ratios relative to $\mathrm{C}_{2} \mathrm{H}$ however show lower values for the PDRs in comparisons with TMC-1, a very likely consequence of the different chemistries at work in respectively illuminated and well-shielded clouds.

PDR model computations (Le Bourlot et al. 1993) using two independent gas-phase chemistry networks succeed in reproducing some of the observed abundances $\left(\mathrm{C}^{18} \mathrm{O}, \mathrm{C}_{2} \mathrm{H}\right)$ and column density ratios $\left(\left[\mathrm{C}^{18} \mathrm{O}\right] /\left[\mathrm{C}_{2} \mathrm{H}\right],\left[c-\mathrm{C}_{3} \mathrm{H}_{2}\right] /\left[\mathrm{C}_{4} \mathrm{H}\right]\right)$. They however fail in producing the observed amount of $c-\mathrm{C}_{3} \mathrm{H}_{2}$ and $\mathrm{C}_{4} \mathrm{H}$ by an order of magnitude. This discrepancy may be to due to incorrect dissociation rates used in the networks, or to missing gas-phase reactions in the considered network. An additional formation process of hydrocarbon could also be at work. We propose to consider further the role of the AIB carriers in the chemistry as their erosion by the UV radiation may return small carbon molecules in the gas phase (see e.g. Allain et al. 1996; Scott et al. 1997; Le Page et al. 2001, 2003). New laboratory experiments (Joblin 2002) confirm that the photodissociation of PAHs can generate various kind of carbon clusters whose further dissociation leads to the release of small fragments, essentially $C_{3}$ and $C_{2}$. Further understanding and implementation of this mechanism in ISM models is needed in the future. More observational data, especially on heavier molecules, would also complete the scarce information on the carbon chemistry of PDRs.

Acknowledgements. We would like to thank the anonymous referee whose comments helped to improve the paper and correct some numerics in the tables. We are also particularly grateful to J. Cernicharo for providing and helping us with his LVG code upgraded with several of the species presented in this paper. We are also grateful to C. Joblin for constructive discussions on the photo-dissociation of PAHs.

\section{References}

Abergel, A., Bernard, J.-P., Boulanger, F., et al. 1996, A\&A, 315, L329 Abergel, A., André, P., Bacmann, A., et al. 1999, in The Universe as Seen by ISO, ed. P. Cox, \& M. F. Kessler, ESA-SP 427

Abergel, A., Bernard, J.-P., Boulanger, F., et al. 2002, A\&A, 389, 239

Abergel, A., Teyssier, D., Bernard, J.-P., et al. 2003, A\&A, 410, 579

Allain, T., Leach, S., \& Sedlmayr, E. 1996, ApJ, 305, 602

Alves, J., Lada, C. J., \& Lada, E. A. 1999, ApJ, 515, 265

Anthony-Twarog, B. J. 1982, AJ, 87, 1213

Barnard, E. E. 1919, ApJ, 49, 1

Bell, M. B., Feldman, P. A., \& Matthews, H. E. 1983, ApJ, 273, L35

Bell, M. B., Feldman, P. A., Watson, J. K. G., et al. 1998, ApJ, 518, 740

Blouin, D., McCutcheon, W. H., Dewdney, P. E., et al. 1997, MNRAS, 287, 455

Bohlin, R. C., Savage, B. D., \& Drake, J. F. 1978, ApJ, 224, 132

Bontemps, S., André, P., Kaas, A. A., et al. 2001, A\&A, 372, 173

Cardelli, J. A., Meyer, D. M., Jura, M., \& Savage, B. D. 1996, ApJ, 467,334

Cernicharo, J., Kahane, C., Gómez-González, J., \& Guélin, M. 1986, A\&A, 167, L5

Cernicharo, J., \& Guélin, M. 1987, A\&A, 176, 299

Cernicharo, J., Cox, P., Fossé, D., \& Güsten, R. 1999, A\&A, 351, 341

Cernicharo, J., Goicoechea, J. R., \& Bénilan, Y. 2002, ApJ, 580, L157

Chastaing, D., Le Picard, S. D., Sims, I. R., \& Smith, I. W. M. 2001, A\&A, 365, 241

Cox, P., Walmsley, C. M., \& Güsten, R. 1989, A\&A, 209, 382

Dickens, J. E., Irvine, W. M., Snell, R. L., et al. 2000, ApJ, 542, 870

Dickens, J. E., Langer, W. D., \& Velusamy, T. 2001, ApJ, 558, 693

Ducati, J. R., Ribeiro, D., \& Rembold, S. B. 2003, ApJ, 588, 344

Emerson, D. T., Klein, U., \& Haslam, C. G. T. 1979, A\&A, 76, 92

Emerson, D. T., \& Gräve, R. 1988, A\&A, 190, 353

Federman, S. R., Strom, C. J., Lambert, D. L., et al. 1994, ApJ, 420, 756 
Fossé, D., Cesarsky, D., Gerin, M., et al. 2000, in Proc. ISO beyond the peaks: the 2nd ISO workshop on analytical spectroscopy, ed. A. Salama, M. F. Kessler, K. Leech, \& B. Schulz, ESA-SP, 456

Fossé, D., Cernicharo, J., Gerin, M., \& Cox, P. 2001, ApJ, 552, 168

Fossé, D. 2003, Ph.D. dissertation, Université Pierre et Marie Curie, Paris, France

Frerking, M. A., Langer, W. D., \& Wilson, R. W. 1982, ApJ, 262, 590

Fuente, A., Martín-Pintado, J., Cernicharo, J., \& Bachiller, R. 1993, A\&A, 276, 473

Fuente, A., Rodrîguez-Franco, A., García-Burillo, S., et al. 2003, A\&A, 406, 899

Gottlieb, C. A., Gottlieb, E. W., \& Thaddeus, P. 1983, ApJ, 264, 740

Green, S., \& Chapman, S. 1978, ApJS, 37, 169

Greene, T. P., \& Young, E. T. 1992, ApJ, 395, 516

Guélin, M., Green, S., \& Thaddeus, P. 1978, ApJ, 224, L27

Guélin, M., Cernicharo, J., Kahane, C., et al. 1987, A\&A, 175, L5

Habart, E., Boulanger, F., Verstraete, L., et al. 2003, A\&A, 397, 623

Habing, H. J. 1968, Bull. Astr. Netherlands, 19, 41

Herbig, G. H. 1996, ARA\&A, 33, 19

Jansen, D. J., van Dishoeck, E. F., \& Black, J. H. 1994, A\&A, 282, 605

Joblin, C. 2002, in Proc. SFChem 2002: Chemistry as a Diagnostic of Star Formation, Waterloo, Ontario, Canada, ed. C. L. Curry, \& M. Fich

Kramer, C., Stutzki, J., \& Winnewisser, G. 1996, A\&A, 307, 915

Kramer, C., Richer, J., Mookerjea, B., et al. 2003, A\&A, 399, 1073

Kulesa, C. A., \& Black, J. H. 2002, in Proc. SFChem 2002: Chemistry as a Diagnostic of Star Formation, Waterloo, Ontario, Canada, ed. C. L. Curry, \& M. Fich

Lada, C. J., Depoy, D. L., Merrill, K. M., \& Gatley, I. 1991, ApJ, 374, 533

Lada, C. J., \& Wilking, B. A. 1980, A\&A, 238, 620

Le Bourlot, J., Pineau des Forêts, G., Roueff, E., \& Flower, D. R. 1993, A\&A, 267, 233

Le Page, V., Snow, T. P., \& Bierbaum, V. M. 2001, ApJS, 132, 233

Le Page, V., Snow, T. P., \& Bierbaum, V. M. 2003, ApJ, 584, 316

Le Petit, F., Roueff, E., \& Le Bourlot, J. 2002, A\&A, 390, 369
Lucas, R., \& Liszt, H. S. 2000, A\&A, 358, 1069

Maddalena, R. J., Moscowitz, J., Thaddeus, P., \& Morris, M. 1986, ApJ, 303, 375

Maier, J. P., Lakin, N. M., Walker, G. A. H., \& Bohlender, D. A. 2001, ApJ, 553, 267

Millar, T. J., Farquhar, P. R. A., \& Willacy, K. 1997, A\&AS, 121, 139

Motte, F., André, P., \& Neri, R. 1998, A\&A, 336, 150

Oka, T., Thorburn, J. A., McCall, B. J., et al. 2003, ApJ, 582, 823

Ossenkopf, V., \& Henning, T. 1994, A\&A, 291, 943

Pety, J., Teyssier, D., Gerin, M., et al. 2003, submitted

Pound, W. M., Reipurth, B., \& Bally, J. 2003, AJ, 125, 2108

Pratap, P., Dickens, J. E., Snell, R. L., et al. 1997, ApJ, 486, 862

Rachford, B. L., Snow, T. P., Tumlison, J., et al. 2002, ApJ, 577, 221

Roueff, E., Felenbok, P., Black, J. H., \& Gry, C. 2002, A\&A, 384, 629

Scott, A., Duley, W. W., \& Pinho, G. P. 1997, ApJ, 489, L193

Shuping, R. Y., Snow, T. P., Crutcher, R., \& Lutz, B. L. 1999, ApJ, 520,149

Spitzer, L. 1978, Physical Processes in the Interstellar Medium (New York: Wiley)

Stepnik, B., Abergel, A., Bernard, J.-P., et al. 2003, A\&A, 398, 551

Takano, S., Masuda, A., Hirahara, Y., et al. 1998, A\&A, 329, 1156

Terzieva, R., \& Herbst, E. 1998, ApJ, 501, 207

Teyssier, D., \& Sievers, A. 1999, A Fast-Mapping Method for Bolometer Array Observations at the IRAM 30-m Telescope, IRAM Technical report

Thaddeus, P., Guélin, M., \& Linke, R. A. 1981, ApJ, 246, L41

Turner, B. E., Herbst, E., \& Terzevia, R. 2000, ApJS, 126, 427

Vakili, F., Granes, P., Bonneau, D., et al. 1984, PASP, 231, 36

van Dishoek, E. F. 1988, in Rate Coefficients in Astrochemistry, 49, ed. T. J. Millar, \& D. A. Williams

Verstraete, L., Pech, C., Moutou, C., et al. 2001, A\&A, 372, 981

Wilking, B. A., \& Lada, C. J. 1983, A\&A, 274, 698

Wilson, T. L., \& Rood, R. 1994, ARA\&A, 32, 191

Wyrowski, F., Schilke, P., \& Walmsley, C. M. 1999, A\&A, 341, 882

Zhou, S., Evans, N. J. II, Koempe, C., \& Walmsley, C. M. 1993, ApJ, 404,232 
D. Teyssier et al.: Carbon budget and carbon chemistry in PDRs, Online Material $p 1$

\section{Online Material}




\section{Appendix A: Column density computations}

The various column densities considered in the study (Sect. 5.1) have been computed under distinct approximations, depending of the species and/or the source. We describe here the assumptions and details of their computation.

\section{A.1. $\mathrm{C}^{18} \mathrm{O}$}

We applied the method described in e.g. Abergel et al. (2003), consisting in using the optically thick emission lines of ${ }^{12} \mathrm{CO}$ to infer the kinetic temperatures in all three sources. The values obtained at the CO emission peaks are indicated in Sect. 5.1. Based on these temperatures, we used a LVG approximation and fitted, when applicable, the $(J=1-0)$ and $(J=2-1)$ transitions of $\mathrm{C}^{18} \mathrm{O}$ with pairs of $\left[n_{\mathrm{H}_{2}}, N\left(\mathrm{C}^{18} \mathrm{O}\right)\right]$. When only one transition was available (e.g. IC 63), an additional assumption was made on the volume density $n_{\mathrm{H}_{2}}$.

\section{A.2. $\mathrm{C}_{2} \mathrm{H}$}

We took advantage from the simultaneous detection of 4 components of the hyperfine structure of the $N=1-0$ transition of $\mathrm{C}_{2} \mathrm{H}$ and applied the so-called HyperFine Structure method (HFS, see e.g.) featured in the CLASS reduction software. At each position, the fit returns the total transition opacity $\tau_{\text {tot }}$, as well as the product $\left[J_{v}\left(T_{\text {ex }}\right)-J_{v}\left(T_{\text {bg }}\right)\right] \times \tau_{\text {tot }}$ (with $T_{\text {bg }}$ the cosmological background temperature). The method gives satisfactory results provided the signal-to-noise ratio is sufficient, and the opacities are not too low $\left(\tau_{\text {tot }}>0.1\right)$.

For optically thin or marginally optically thick lines, the column density is directly proportional to the integrated line intensity. For the excitation temperature ranges inferred in the three objects, $N\left(\mathrm{C}_{2} \mathrm{H}\right)$ is not sensitive to $T_{\mathrm{ex}}$, and we finally adopted the following expressions $\left(\right.$ in $\mathrm{cm}^{-2}$ ):

$N\left(\mathrm{C}_{2} \mathrm{H}\right)=(7 \pm 1) \times 10^{13} \times W\left(\mathrm{C}_{2} \mathrm{H}\right) / G(\tau)$

where $G(\tau)=\left(1-\mathrm{e}^{-\tau^{\prime}}\right) / \tau^{\prime}, \tau^{\prime}=0.679 \times \tau^{0.911}$, is a correction factor accounting for non-negligible opacity corrections (Wyrowski et al. 1999), and $\tau$ is the opacity of the main line at $87.316 \mathrm{GHz}$.

\section{A.3. $c-\mathrm{C}_{3} \mathrm{H}_{2}$}

Based on LVG simulations, we found that the $c-\mathrm{C}_{3} \mathrm{H}_{2}$ intensities can be very sensitive to density variations in the conditions encountered in our targets $\left(1-5 \times 10^{4} \mathrm{~cm}^{-3}, T_{\text {kin }} \simeq 20-50 \mathrm{~K}\right)$. As an illustration, considering the Horsehead case, the density uncertainties derived from the $\mathrm{C}^{18} \mathrm{O}$ analysis would lead to $N\left(c-\mathrm{C}_{3} \mathrm{H}\right)$ variations larger of $50 \%$. To solve this problem, we used the good correlation observed between the brightness temperatures of $c-\mathrm{C}_{3} \mathrm{H}_{2}$ and $\mathrm{C}_{2} \mathrm{H}$ (e.g. Fig. 7). Since $\mathrm{C}_{2} \mathrm{H}$ is much less affected by density variations, and considering the low optical depth of the molecules, this trend suggests homogeneous physical conditions in the clouds, although the excitation of each species strongly differs. We thus assumed a constant volume density and a representative kinetic temperatures to apply to the LVG simulations. Since our LVG code treats ortho and para forms separatly, we applied to the ortho $c-\mathrm{C}_{3} \mathrm{H}_{2}\left(2_{1,2}-1_{0,1}\right)$ transition a standard $o / p$ ratio of 3 .

\section{A.4. $\mathrm{C}_{4} \mathrm{H}$}

The low line intensities suggest very likely optically thin lines. Applying the same reasoning as for $c-\mathrm{C}_{3} \mathrm{H}_{2}$, we performed LVG simulations on the $\mathrm{C}_{4} \mathrm{H}(9-8)$ transition lines. Since the model does not account for the hyperfine structure of this transition, we built synthetic lines from the emission of all observed components. This combination uses the expected (at LTE) relative line intensity ratios $\alpha_{N}$, and writes:

$W_{\text {synth }}=\frac{\Sigma\left(W_{N} / \alpha_{N}\right)}{N}$

where $N$ is the nth hyperfine component. In practice, the relative intensities of the $(N=9-8, \mathrm{~J}=19 / 2-17 / 2$, hereafter labelled 1) and ( $N=9-8, J=17 / 2-15 / 2$, labelled 2$)$ transitions (consisting both of two blended lines) are 0.53 and 0.47 respectively, so that $W_{\text {synth }} \simeq W_{1}+W_{2}$. The outputs of the LVG simulations are given in Table 2 .

\section{A.5. Other species}

The emissions exhibited by the rarer molecules introduced in Sect. $3\left(l-\mathrm{C}_{3} \mathrm{H}, c-\mathrm{C}_{3} \mathrm{H}, l-\mathrm{C}_{3} \mathrm{H}_{2}, l-\mathrm{C}_{4} \mathrm{H}_{2}, \mathrm{CH}_{3} \mathrm{C}_{2} \mathrm{H}, \mathrm{C}_{6} \mathrm{H}\right.$ and $\mathrm{HC}_{3} \mathrm{~N}$ ) have been analysed under LTE assumptions, using the densities and excitation temperatures inferred from the analyses described previously. In particular, for $c-\mathrm{C}_{3} \mathrm{H}$ and $l-\mathrm{C}_{3} \mathrm{H}$ we used the $T_{\text {ex }}$ derived from $\mathrm{C}_{2} \mathrm{H}$. For $\mathrm{C}_{6} \mathrm{H}$, there are no cross sections available. We estimated them from those of $\mathrm{HC}_{3} \mathrm{~N}$ (Green \& Chapman 1978, - see Cernicharo et al. 1999). Finally, for $\mathrm{HC}_{3} \mathrm{~N}$, we assumed the $T_{\text {kin }}$ values inferred in Sect. A.1. In case of non-detection, upper limits of the column densities are given (Table 2).

\section{A.6. $\mathrm{H}_{2}$ column densities}

Meaningful comparisons of the observed molecular column densities require an accurate estimate of the total $\mathrm{H}_{2}$ column densities at each of the positions reported in Table 2. We discuss here the methods used to derive as accurate as possible values for each of our targets.

\section{A.6.1. L1688-W}

In this source, we cannot use the extinction map of Wilking \& Lada (1983) as our positions lie outside their coverage. We nevertheless reproduced their approach, consisting in converting $\mathrm{C}^{18} \mathrm{O}$ column density into visual extinction, and applied the $N\left(\mathrm{H}_{2}\right) / A_{\mathrm{V}}$ calibration by Bohlin et al. (1978), which assumes a gas-to-dust ratio of 100 . The choice of the the $A_{\mathrm{V}} / N\left(\mathrm{C}^{18} \mathrm{O}\right)$ ratio is not straightforward as most reported values have been obtained in cold dark clouds where depletion onto grains affects the measurements and consequently increase this ratio (e.g. Frerking et al. 1982; Cernicharo et al. 1987). One can however recompute this ratio for warmer conditions assuming that all $\mathrm{CO}$ is in the gas phase. Using high-resolution infrared 
spectroscopy of $\mathrm{H}_{2}$ and CO in NGC 2024 IRS2, Kulesa et al. (2002) recently reported a new measurement of the $\mathrm{CO}$ abundance relative to $\mathrm{H}_{2}$, $[\mathrm{CO}]=3 \times 10^{-4}$. With $\left[\mathrm{O}^{16}\right] /\left[\mathrm{O}^{18}\right]=560$ (Wilson \& Rood 1994) and using standard conversion numbers, one can infer:

$A_{\mathrm{V}} / N\left(\mathrm{C}^{18} \mathrm{O}\right)=R \times 6.6 \times 10^{-16} \mathrm{mag} \mathrm{cm}{ }^{2}$

where $R=A_{\mathrm{V}} / E(B-V)$ has reported values ranging between 3-5 (Spitzer 1978; Ducati et al. 2003 and ref. therein). This yields:

$A_{\mathrm{V}} / N\left(\mathrm{C}^{18} \mathrm{O}\right)=2-3.3 \times 10^{-15} \mathrm{mag} \mathrm{cm}^{2}$

in very good agreement with the ratio proposed by Wilking \& Lada (1983). A zero point offset in $A_{\mathrm{V}}$ is however to be considered to take into account the fact that $\mathrm{C}^{18} \mathrm{O}$ is photo-dissociated for low extinctions. We used an offset of $1.5 \mathrm{mag}$, consistent with the calibrations by Frerking et al. (1982) and Cernicharo et al. (1987). We finally adopted the following conversion rule for L1688-W:

$N\left(\mathrm{H}_{2}\right)=(2.5 \pm 0.6) \times 10^{6} \times N\left(\mathrm{C}^{18} \mathrm{O}\right)+1.4 \times 10^{21} \mathrm{~cm}^{-2}$

At the peak $\mathrm{C}^{18} \mathrm{O}$ column density, this yields $N\left(\mathrm{H}_{2}\right)=1.1 \times$ $10^{22} \mathrm{~cm}^{-2}$.

\section{A.6.2. Horsehead nebula}

In the Horsehead nebula, no extinction estimate is available at spatial resolution of order $30^{\prime \prime}$. With a beam resolution of $\sim 2^{\prime}$, Kramer et al. (1996) report $A_{\mathrm{V}} \sim 3$. This is consistent with rough estimates from the IRAS $100 \mu \mathrm{m}$ emission ( $4^{\prime}$ beam) but is very likely not adapted to the small-scale structure of this source. Applying the same approach as in L1688-W implies extinctions of order 8-13 mag. In order to cross-check these estimates, we have used our observations of the continuum at $1.2 \mathrm{~mm}$ (Fig. 4) and exploited them in the fashion described by Motte et al. (1998), with:

$N_{\mathrm{H}_{2}}=\frac{S_{1.2 \mathrm{~mm}}}{\Omega_{\text {beam }} \mu m_{\mathrm{H}} \kappa_{1.2} B_{1.2}\left(T_{\text {dust }}\right)}$ where $S_{\mathrm{mm}}$ is the $1.2 \mathrm{~mm}$ flux density in mJy per $11^{\prime \prime}$ beam, $\Omega_{\text {beam }}$ the beam solid angle, $\mu$ the mean molecular weight, $m_{\mathrm{H}}$ the mass of atomic hydrogen, $B_{1.2}(T)$ the Planck function at wavelength $1.2 \mathrm{~mm}$ and temperature $T$, and $\kappa_{1.2}$ the dust mass opacity at $1.2 \mathrm{~mm}$. Such estimates are affected by the approximative knowledge of the dust properties at these wavelengths (e.g. Ossenkopf \& Henning 1994; Stepnik et al. 2003). With the additional assumption that $T_{\text {dust }}$ is well represented by $T_{\text {kin }}\left(\simeq 40 \mathrm{~K}\right.$ here), and adopting $\kappa_{1.2}=3-6 \times 10^{-3} \mathrm{~g} \mathrm{~cm}^{-2}$, we derived from the observed peak flux of $56 \mathrm{mJy} /$ beam a peak extinction of 12-25 mag. After smoothing to the $3 \mathrm{~mm}$ beam, the corresponding extinction range reduces to $9-15 \mathrm{mag}$, which translate into a column density of $N\left(\mathrm{H}_{2}\right)=1.1 \pm 0.3 \times$ $10^{22} \mathrm{~cm}^{-2}$. This is significantly above any previous estimates (cf. Sect. 2.1), but in very good agreement with the independent calculation using $\mathrm{C}^{18} \mathrm{O}$ emission.

\section{A.6.3. IC 63}

In IC 63 , we simply used the value adopted by Jansen et al. (1994) $N\left(\mathrm{H}_{2}\right)=5 \pm 2 \times 10^{21} \mathrm{~cm}^{-2}$, although it may be overestimated at positions away from the $\mathrm{CO}$ peak. 